\title{
Miniscope-LFOV: A large field of view, single cell resolution, miniature microscope for wired and wire-free imaging of neural dynamics in freely behaving animals
}

Changliang Guo ${ }^{1,2,4,8, *}$, Garrett J. Blair,3, ${ }^{3, *}$, Megha Sehgal ${ }^{3,4,5,6}$, Federico N. Sangiuliano Jimka ${ }^{1,2,4,8}$, Arash Bellafard $^{1,2}$, Alcino J. Silva ${ }^{3,4,5,6}$, Peyman Golshani ${ }^{1,2,4,5,7,8,10,11}$, Michele A. Basso ${ }^{1,5,6,7,8}, \mathrm{H}$. Tad Blair ${ }^{3}$, and Daniel Aharoni $i^{1,2,4,8, \#}$

*These authors contributed equally.

${ }^{1}$ David Geffen School of Medicine, University of California Los Angeles, Los Angeles, CA, 90095 USA ${ }^{2}$ Department of Neurology, David Geffen School of Medicine, University of California, Los Angeles, Los Angeles, CA, USA

${ }^{3}$ Department of Psychology, UCLA, Los Angeles, CA 90095-1563, USA.

${ }^{4}$ Integrative Center for Learning and Memory, University of California, Los Angeles, Los Angeles, CA, USA

${ }^{5}$ Department of Psychiatry and Biobehavioral Sciences, University of California, Los Angeles, CA 90095.

${ }^{6}$ Department of Neurobiology, University of California, Los Angeles, CA 90095.

${ }^{7} J a n e$ and Terry Semel Institute for Neuroscience and Human Behavior, University of California, Los Angeles, CA 90095 USA

${ }^{8}$ Brain Research Institute, University of California, Los Angeles, CA 90095.

${ }^{9}$ Department of Physiology, David Geffen School of Medicine, University of California, Los Angeles, Los Angeles, CA, USA.

${ }^{10}$ West LA Veterans Affairs Medical Center, Los Angeles, CA, USA.

${ }^{11}$ Intellectual and Developmental Disabilities Research Center, University of California, Los Angeles, Los Angeles, CA, USA.

${ }^{\#}$ Correspondence should be addressed to Daniel Aharoni (daharoni@mednet.ucla.edu)

\section{Abstract:}

We present a large field of view (FOV) open-source miniature microscope (MiniLFOV) designed to extend the capabilities of the UCLA Miniscope platform to large-scale, single cell resolution neural imaging in freely behaving large rodents and head-fixed mice. This system is capable of multiple imaging configurations, including deep brain imaging using implanted optical probes and cortical imaging through cranial windows. The MiniLFOV interfaces with existing open-source UCLA Miniscope DAQ hardware and software, can achieve single cell resolution imaging across a $3.6 \times 2.7 \mathrm{~mm}$ field of view at 23 frames per second, has an electrically adjustable working distance of up to $3.5 \mathrm{~mm} \pm 150 \mu \mathrm{m}$ using an onboard electrowetting lens, incorporates an absolute headorientation sensor, and weighs under 14 grams. The MiniLFOV provides a 30-fold larger FOV and yields 20-fold better sensitivity than Miniscope V3, and a 12-fold larger FOV with 2-fold better sensitivity than Miniscope V4. Power and data transmission are handled through a single, flexible coaxial cable down to $0.3 \mathrm{~mm}$ in diameter facilitating naturalistic behavior. We validated the MiniLFOV in freely behaving rats by simultaneously imaging >1000 GCaMP7s expressing neurons in the CA1 layer of the hippocampus and in head-fixed mice by simultaneously imaging $\sim 2000$ neurons in the mouse dorsal cortex through a $4 \times 4 \mathrm{~mm}$ cranial window. For freely behaving experiments, the MiniLFOV supports optional wire-free operation using a $3.5 \mathrm{~g}$ wire-free data acquisition expansion board which enables close to 1-hour of wire-free recording with a $400 \mathrm{mAh}(7.5 \mathrm{~g}) \mathrm{on}-$ board single-cell lithium-polymer battery and expands wire-free imaging techniques to larger animal models. We expect this new open-source implementation of the UCLA Miniscope platform will enable researchers to address novel hypotheses concerning brain function in freely behaving animals. 
Understanding how populations of neurons and underlying circuits give rise to complex behavior remains a key endeavor of systems neuroscience. Novel imaging tools combined with the development of new calcium $\left(\mathrm{Ca}^{2+}\right)$ indicators have advanced neuroscience by introducing the ability to monitor large populations of neurons simultaneously and track identified neurons across weeks to months ${ }^{1}$. Decades of neuroscientific research has led to the development and refinement of optical techniques, such as two-photon microscopy ${ }^{2-7}$, three-photon microscopy $^{8}$, confocal microscopy ${ }^{9,10}$, and light sheet microscopy ${ }^{11,12}$ to image the structures and functions of large neuronal networks with cellular and subcellular resolutions ${ }^{13}$. However, many of these approaches require bulky imaging devices that can only be used on head restrained animals ${ }^{14,15}$, constraining experiments from being conducted in more naturalistic environments and behaviors.

Single photon epifluorescence miniature microscopy ${ }^{16,17}$ and multi-photon miniature microscopy ${ }^{18-21}$ circumvent $^{2}$ the constraint of head stabilization while still achieving single cell resolution, enabling these optical techniques in freely behaving animals and expanding the repertoire of behavioral assays that can be used in conjunction with neural imaging. Open-source head-mounted miniaturized epifluorescence microscopes, such as the opensource UCLA Miniscope ${ }^{22-25}$, FinchScope ${ }^{26}$, miniScope ${ }^{27,28}$, NiNscope ${ }^{29}$, CHEndoscope ${ }^{30}$, MiniLFM $^{31}$, Miniscope $3 \mathrm{D}^{32}$, and counterpart miniscopes ${ }^{33-36}$, have shown that wide-field epifluorescence imaging can be achieved with miniaturized microscopes that are light enough to be mounted on the head of a rodent and record behaviorally relevant neural signals that can be extracted by further analytical techniques ${ }^{37-40}$. These developments over the past decade have been used extensively to conduct neural imaging in freely behaving animals to reveal neural dynamics related to learning and memory ${ }^{22}$, neurological disorders ${ }^{25}$, and even social interactions ${ }^{28}$.

Previous designs of UCLA Miniscopes were developed specifically for small animals, such as mice, which resulted in a field of view (FOV) of no more than $0.8 \mathrm{~mm}^{2}$, limiting their capabilities and applications in imaging large-scale, single cell fluorescent dynamics ${ }^{41,42}$ in larger animals. Furthermore, $\mathrm{Ca}^{2+}$ indicators generally have lower expression and fluorescence in animals other than mice, motivating the need for a high-sensitivity, large FOV imaging system with single cell resolution. In addition, current wired miniature microscopy devices lack optional configurations for wire-free operation, impeding experiments that involve recording multiple interacting animals simultaneously or animals navigating large or vertically confined environments ${ }^{43-49}$.

Here we report a head-mounted, open-source Large Field of View Miniscope (MiniLFOV) developed as a new implementation of the UCLA Miniscope platform. It has two optical configurations optimized at different working distances (WD) for superficial and deep neural imaging. A 1.8-mm-WD configuration provides $2.5 \mu \mathrm{m}$ (center) to $4.4 \mu \mathrm{m}$ (edge) resolution across $3.1 \times 2.3 \mathrm{~mm}$ FOV, and a 3.5-mm-WD enables $3.5 \mu \mathrm{m}$ (center) to $6.2 \mu \mathrm{m}$ (edge) resolution across $3.6 \times 2.7 \mathrm{~mm}$ FOV. With a high sensitivity 5MP monochrome complementary metaloxide-semiconductor (CMOS) image sensor (MT9P031, Onsemi) with up to 8x analog gain, the MiniLFOV yields 20-fold better sensitivity than the previous generation Miniscope V3, and twice the sensitivity compared to current generation Miniscope V4. The WD is electrically adjustable to cover $1.8 \mathrm{~mm} / 3.5 \mathrm{~mm} \pm 150 \mu \mathrm{m}$ depth focus using an electrowetting lens (EWL; Corning Arctic 58N, Varioptics/Corning). Incorporated with a 9-axis absoluteorientation sensor (BNO055, Bosch), the MiniLFOV has the ability to collect head movement data as absolute orientation quaternions, Euler angles, and linear acceleration at up to $100 \mathrm{~Hz}$, which can help investigate head orientation related neural mechanisms ${ }^{50-53}$.

The MiniLFOV is $35 \mathrm{~mm}$ tall, weighs 13.9 grams, and can be easily modified for different experiments by swapping its custom objective module. The two WD configurations enable multiple applications such as cortical imaging through a cranial window and deep brain imaging using one or multiple implanted optical probes such as a relay Gradient Refractive Index (GRIN) lens. Furthermore, we also developed a small, wearable wire-free data acquisition (DAQ) expansion board (wire-free DAQ, $3.5 \mathrm{~g}$ ) which adds wire-free capabilities to all generations of coaxial cabled UCLA Miniscopes. The wire-free DAQ is battery powered by an on-board, singlecell lithium-polymer battery and records imaging data onto an on-board microSD card. The wire-free DAQ can be mounted directly onto the MiniLFOV's housing or be worn as a backpack. Power, bi-directional configuration commands, and high bandwidth uni-directional imaging data are transmitted between the MiniLFOV and wirefree DAQ via a short coaxial cable. Integrated with an IR remote control receiver, the system supports starting, stopping, and pausing recordings remotely with an IR remote control transmitter during experiments. 
We validated the wired MiniLFOV in freely behaving rats running on a large rectangular track by imaging GCaMP7s expressing neurons in the hippocampal CA1 pyramidal layer using two $1.8 \mathrm{~mm}$-diameter quarter pitch GRIN lenses stacked together $\left(F O V=\sim 3.14 \mathrm{~mm}^{2}\right.$ ) with over 1,300 cells imaged in a single 15-minute session. We also validated the wire-free configuration of the MiniLFOV in rats exploring an open circular field. In addition, we validated the MiniLFOV in head-fixed mice on a circular treadmill by imaging GCaMP6f expressing neurons in dorsal cortex through a cranial window with over 1,900 neurons recorded in a single 7-minute session. With greater than a 30-fold increase in FOV than the previous generation UCLA Miniscope V3 and 12-fold increase in FOV than the current generation UCLA Miniscope V4 (Video 1), we expect this imaging platform will generate qualitatively and quantitatively new data for neuroscience applications in freely behaving large animals. As larger animals, rats, for example, can perform more sophisticated behaviors (such as autism spectrum disorder, addictive behavior, cognition, etc $)^{54}$, increasing the yield of neurons recorded simultaneously continues to yield novel discoveries about brain function, particularly when activity is encoded within a subset of the population ${ }^{55-}$ 59 , by adopting tools that are specifically designed for them. Importantly, the MiniLFOV is fully open-source, building off the already widely adopted open-source UCLA Miniscope ecosystem, and has been developed to reduce the technical and economic hurdles common in novel tool adoption, making it broadly accessible to the global neuroscience community.

\section{Results}

\section{System design}

The MiniLFOV (Fig. 1a) consists of three modules, an objective module, an emission module, and a sensor module, holding the optical components and custom Rigid-Flex printed circuit board (PCB). The 3D module bodies are designed in Autodesk Fusion 360 (educational license) and printed with black resin (Formlabs, FLGPBK04) by a table-top stereolithographic (SLA) 3D printer (Formlabs, Form 3) to produce a lightweight, compact, and low-cost assembly. The circuit schematic and Rigid-Flex PCB layout are designed using KiCad, a free software suite for electronic design automation (EDA). The circuit module consists of 4 sub-circuits, an excitation LED circuit, an electrowetting lens tuning and head orientation circuit, a CMOS image sensor circuit for collecting $\mathrm{Ca}^{2+}$ imaging data, and a Power-over-coax and serializer circuit (Fig. 1a, b) for supporting coaxial cable power and data transmission. These four sub-circuits are connected by an embedded flex printed circuit without any additional soldering required.

The 1.8-mm-WD objective module (Fig. 1c) contains 3 achromatic lenses (\#45-345, \#49-656, \#45-092, Edmund Optics) and a 3D printed spacer. The emission module (Fig. 1d) holds the excitation filter $(4 \times 4 \times 1.1 \mathrm{~mm}$, ET470/40x, Chroma), dichroic mirror (14 × $10 \times 1 \mathrm{~mm}$, T500spxr, Chroma), emission filter $(10 \times 10 \times 1 \mathrm{~mm}$, ET525/50m, Chroma), aspherical lens (\#49-658, Edmund Optics), 3D printed lens holder, and concave lens (\#45-019, Edmund Optics). The sensor module is designed for holding the electrowetting lens (Corning Arctic $58 \mathrm{~N}$, Varioptics/Corning) driven by an EWL driver (MAX14515, Maxim) and a 5MP monochrome CMOS image sensor (MT9P031, Onsemi), see Figure 1b. The main modules are screwed together with two types of screws, M1 thread-forming screws and 0-80 socket head screws.

Power, communication, and image data transmission are packed into a single, flexible $50 \Omega$ coaxial cable (CW2040-3650SR, Cooner Wire) using power-over-coax filtering and a serializer/deserializer pair for bidirectional control communication and uni-direction high bandwidth data streaming (TI DS90UB913A/DS90UB914A). For coaxial cable lengths longer than 2.5 meters, an external $6 \mathrm{~V}$ power supply should be connected to the UCLA Miniscope DAQ, replacing the power supplied by the USB connection to the MiniLFOV. With a total cable diameter down to $0.3 \mathrm{~mm}$ (Molex 100065-0023) and compatibility with active and passive, low torque commutators, this design minimizes the impact of cabling on animal behavior.

The MiniLFOV interfaces with the open-source Miniscope DAQ Software to stream, visualize, and record neural dynamics and behavioral data. The software enables multiple Miniscope and behavioral camera video streams, allowing for multi-animal neural and behavioral recordings. The DAQ hardware and software used for the MiniLFOV are the same as what has been used for all other UCLA Miniscope devices ${ }^{22}$. This DAQ platform allows for LED brightness adjustment, image sensor gain adjustment, focus adjustment by the EWL, frame rate selection, real-time $\Delta F / F$ and trace visualization, and works with embedded DLC-Live ${ }^{60}$ for real-time pose 

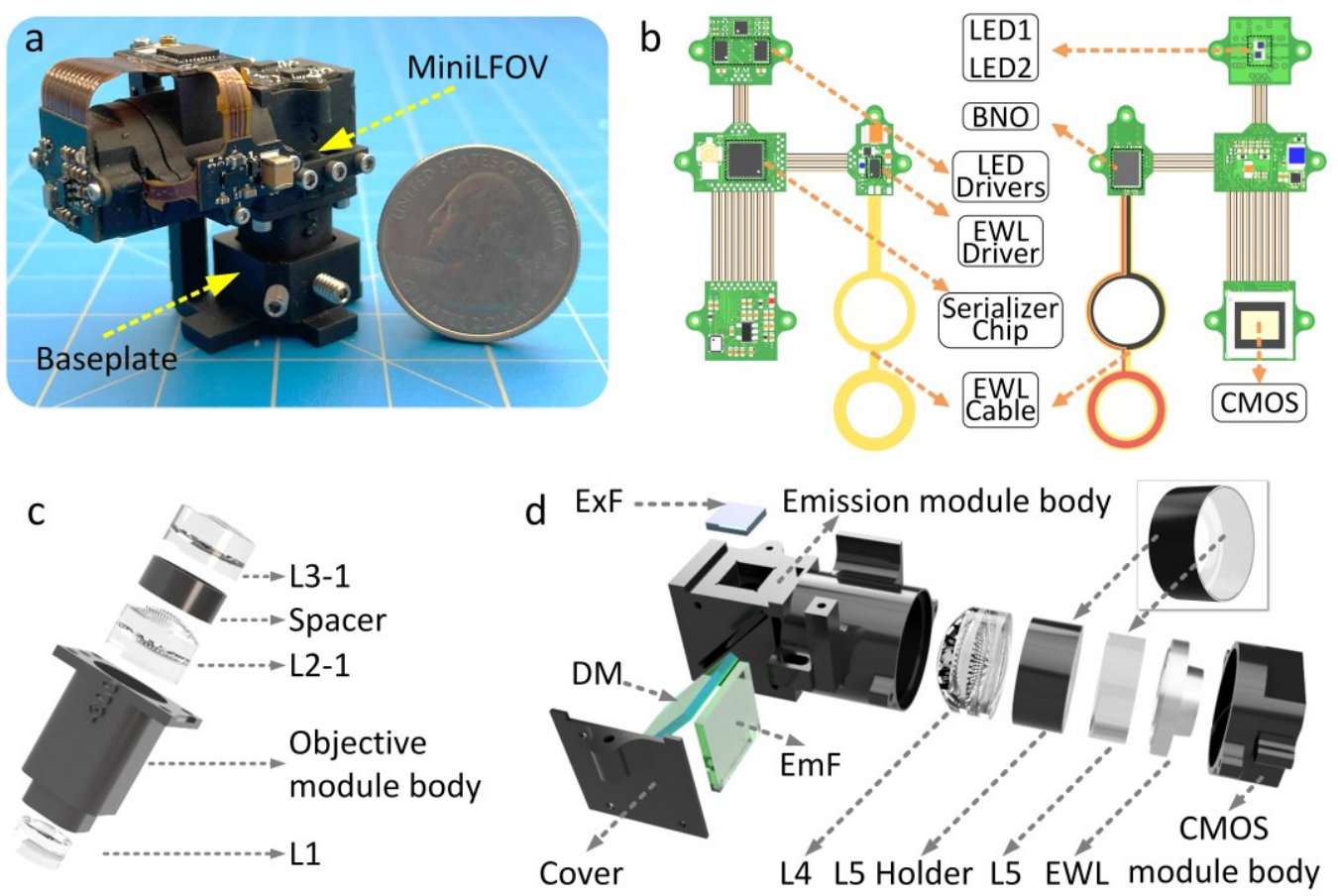

Figure 1. Design of MiniLFOV. (a) Photograph of MiniLFOV with baseplate. (b) Rigid-Flex PCB of the MiniLFOV consisting of four rigid $\mathrm{PCBs}$ connected by an internal flex printed circuit. Two LEDs are housed on the LED circuit board and driven by two led drivers with an $\mathrm{I}^{2} \mathrm{C}$ digital potentiometer for brightness control. An EWL driver and EWL cable holding the EWL is housed along side an absolute-orientation sensor (BNO055) for collecting head orientation data. A 5MP monochromatic CMOS image sensor (MT9P031) is used for capturing $\mathrm{Ca}^{2+}$ fluorescence and sending digitized image data to the serializer system. A serializer chip serializes the imaging data and sends it over a coaxial connector to communicate with a custom MiniscopeDAQ system. (c) Objective module of the MiniLFOV (1.8-mm-WD configuration) containing a 3D printed objective module body, three achromatic lenses (L1, L2-1, and L31) and a spacer between L2-1 and L3-1. (d) Emission and sensor module of the MiniLFOV. The emission module consists of a 3D printed emission module body with a 3D printed cover, aspheric lens L4, a plane-concave lens L5, L5 holder, excitation filter (ExF), dichroic mirror (DM), and emission filter (EmF). The sensor module consists of a 3D CMOS module body holding the Electrowetting lens (EWL) and a mounting pocket for the CMOS image sensor.

estimation. The DAQ supports synchronizing with external devices through an outputted frame synchronization signal (toggles a 3.3 V signal high and low on every frame acquisition) or an input trigger to externally trigger the recording. The list of the components used are given in Table 2.

MiniLFOV accessories also include a reinforced baseplate attached to the skull with dental/bone cement which allows consistent mounting of the MiniLFOV, and a protective cap to cover the GRIN lenses implanted. Details of assembling the MiniLFOV are given in Methods section and Figure s1.

\section{Optical performance}

The optics of the system was designed and optimized using Zemax OpticsStudio. The excitation path (blue in Fig. 2a) and emission path (green in Fig. 2a) are separated by the dichroic mirror (T500spxr, Chroma), which also folds the emission path to lower the center of mass of the system (Fig. 2a, e and $\mathbf{g}$ ). The emission filter (ET525/50m, Chroma) is placed in the emission path to block possible excitation light leakage and back-scatter. The high resolution of the system and large FOV are achieved by using 5 off-the-shelf lenses from Edmund Optics (L1, L2-1/L2-2, L3-1/L3-2, L4, and L5), with optimized distance and sequence between them. Achromatic lenses are adopted to minimize chromatic aberrations, enabling color-sequential imaging and customization for different fluorophores. The 1.8-mm-WD configuration of the system is designed to have a $0.25 \mathrm{NA}$ at the object space and a magnification of 1.9 (Fig. 2d, e). As shown in the spot diagram (Fig. 2e) at the image plane, the Modulation Transfer Function (MTF) curves reach zero at $500 \mathrm{lps} / \mathrm{mm}(2 \mu \mathrm{m})$. The root-mean-square (RMS) radius of the on-axis spot $(x, y=0 \mathrm{~mm})$ is $1.2 \mu \mathrm{m}$ and the off-axis spots $(x=0, y=1.5 \mathrm{~mm} ; \mathrm{x}=0, y=-1.5 \mathrm{~mm})$ are $2.3 \mu \mathrm{m}$, fitting the requirement for resolving single neuronal bodies in the brain. The field curvature of the optics 
bioRxiv preprint doi: https://doi.org/10.1101/2021.11.21.469394; this version posted November 22, 2021. The copyright holder for this preprint (which was not certified by peer review) is the author/funder, who has granted bioRxiv a license to display the preprint in perpetuity. It is made available under aCC-BY-NC-ND 4.0 International license.

in objective space across a $3 \mathrm{~mm}$ FOV is $130 \mu \mathrm{m}$. The optical system also enables an electrically adjustable WD of $\pm 150 \mu \mathrm{m}$ in the object space with the EWL placed before the CMOS image sensor (Fig. 1d). To collect the large FOV at its highest resolution, a CMOS image sensor is used with $2592 \times 1944$ pixels of $2.2 \mu \mathrm{m}$ pixel size.
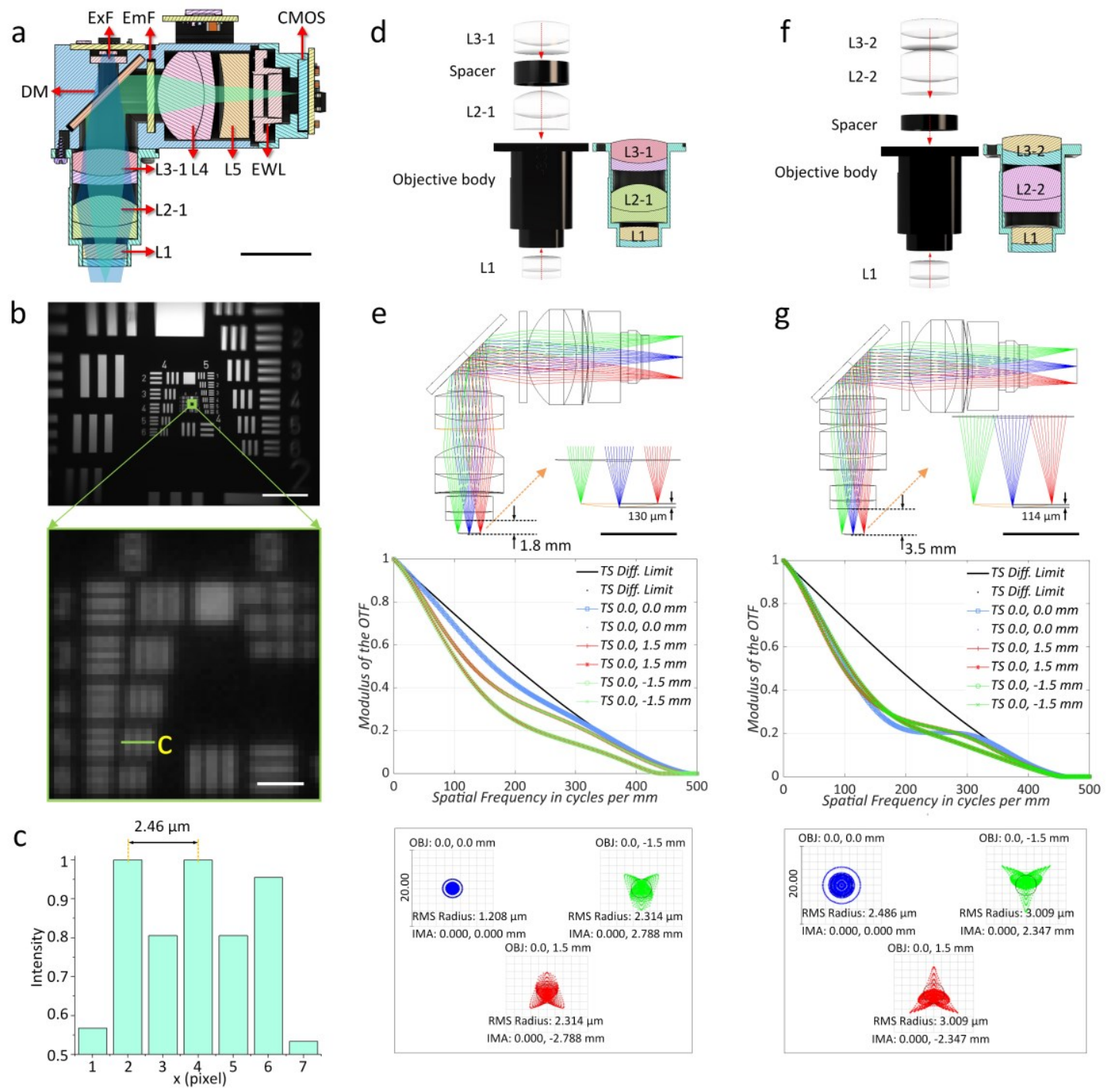

Figure 2. Optical design and performance of the MiniLFOV. (a) Cross section of the MiniLFOV assembly, showing the optical components inside the body, including objective module (1.8-mm WD) with 3 achromatic lenses (L1, L2-1, and L3-1), emission module with 1 achromatic lens (L4), 1 concave lens (L5), and 1 electrowetting lens (EWL). It also includes excitation filter (ExF), Dichroic mirror (DM), and emission filter $(\mathrm{EmF})$. Excitation path from 2 LEDs is highlighted in blue and emission path is highlighted in green. Scale bar: $10 \mathrm{~mm}$. (b) The $3.1 \times 2.3 \mathrm{~mm}$ FOV is shown using a Negative USAF 1951 Hi-Resolution Target (\#55-622, Edmund Optics). Scale bar: $500 \mu \mathrm{m}$ (top), and $10 \mu \mathrm{m}$ (bottom). (c) $2.46 \mu \mathrm{m}$ or $406 \mathrm{lps} / \mathrm{mm}$ can be resolved experimentally from the cross-sectional profile along the green line in (b, bottom). (d) 1.8-mm-WD objective module configuration is shown for use in deep braining imaging. (e) Zemax simulation of emission path shows the $1.85 \mathrm{~mm}$-WD-configuration with $130 \mu \mathrm{m}$ field cuvature in the object space (top), MTF of the three positions on the image plane (middle), and spot diagram of the optics (bottom). The values of this function reach zero when $x$ gets to $500 \mathrm{lps} / \mathrm{mm}$, i.e. $2 \mu \mathrm{m}$ resolution, at the image plane (1.1 $\mu \mathrm{m}$ in the object space), which closely agrees with measured results of the system. As shown in the spot diagram of the optics at the image plane, RMS radius is $1.208 \mu \mathrm{m}$ at the center and $2.134 \mu \mathrm{m}$ at $(0,1.5 \mathrm{~mm} /-1.5 \mathrm{~mm})$. The magnification of the optics is given by $2.788 / 1.5=1.86 \mathrm{calculated}$ from the spot diagram, see (e, bottom), in which the points $(0,1.5 \mathrm{~mm} /-1.5 \mathrm{~mm})$ are focused at $(0,2.788 \mathrm{~mm} /-2.788 \mathrm{~mm})$ on the CMOS sensor. (f) 3.5-mm-WD objective module configuration is shown for use in cortical imaging through a cranial window. It is configured by using the same L1 lens, but uses two different achromatic lenses, L2-2 and L3-2, with a spacer placed between L1 and L2-2. (g) ) Zemax simulation of emission path shows the 3.5-mm-WD configuration with $114 \mu \mathrm{m}$ field curvature in the object space (top). MTF curves reach zero when $x$ axis gets $450 \mathrm{lps} / \mathrm{mm}$, i.e. $2.2 \mu \mathrm{m}$ at the image plane (1.4 $\mu \mathrm{m}$ in the object space) (middle). 3.5-mm WD (right) has RMS radius $2.486 \mu \mathrm{m}$ at the center and $3.009 \mu \mathrm{m}$ at $(0,-1.5 \mathrm{~mm} / 1.5 \mathrm{~mm})$. The magnification is calculated by $2.347 / 1.5=1.56$ (bottom), with $3.6 \mathrm{~mm} \times 2.7 \mathrm{~mm}$ FOV achivable. experimentally. 
As the FOV is slightly constrained by the vertical pixel height of the sensor, the final FOV achievable is $3.1 \mathrm{~mm}$ $\times 2.3 \mathrm{~mm}$, validated by imaging a Negative USAF $1951 \mathrm{Hi}-$ Resolution Target (\#55-622, Edmund Optics) (Fig. 2b). In practice, the actual spatial resolution of the system is constrained by the pixel size of this image sensor and the magnification of the system, giving $2.46 \mu \mathrm{m}$ (Group 8 Element 5, green box), or $406 \mathrm{lps} / \mathrm{mm}$ resolution (Fig. 2b, c) at the center of the FOV and dropping to $4.4 \mu \mathrm{m}$ at the edge. Driven with a $66.667 \mathrm{MHz}$ pixel clock, the sensor runs at 11 frames per second (FPS) at full resolution and 23 FPS with $2 x$ horizontal and vertical binning. With 2x pixel binning, $4.4 \mu \mathrm{m}$ resolution can be achieved at the center of the FOV, dropping to $6.2 \mu \mathrm{m}$ at the edge and is the configuration used for all data collection shown in this paper. For higher FPS (up to 15 FPS at full resolution and 30 FPS at 2x binned resolution), a $96 \mathrm{MHz}$ pixel clock can be used but requires shorter or larger diameter coax cables for stable use. Additionally, faster frame rates can be achieved by cropping the recording window to the needed size or increasing the binning factor. To optically excite large FOVs to activate $\mathrm{Ca}^{2+}$ indicators, two LUXEON Z Blue LEDs (470 nm, LXZ1-PB01) are available in the excitation path with a band-pass excitation filter (ET470/40x, Chroma) placed below the LEDs to narrow their spectral band. With the highly sensitive CMOS image sensor (MT9P031, Onsemi), the MiniLFOV after $2 \times 2$ pixel binning and maximum analog gain (MiniLFOV_bin2x; gain=8x) has twice the sensitivity than that of a Miniscope V4 at maximum analog gain (gain=3.5x) resulting in a $2 x$ decrease in excitation power needed per unit area. A comparison of the sensitivity of Miniscope V3, Miniscope V4, MiniLFOV with full resolution (MiniLFOV_full), and MiniLFOV after 2 $\times 2$ pixel binning (MiniLFOV_bin2x), is shown in Figure s2.

The 3.5-mm-WD configuration (Fig. 2f, g) uses L1, L2-2 (\#49-657, Edmund Optics), and L3-2 (\#45264, Edmund Optics) in the objective module and is designed to give additional space below the bottom of the MiniLFOV for imaging through cranial windows or other thick samples. MTF curves drop to zero at $450 \mathrm{lps} / \mathrm{mm}(2.2 \mu \mathrm{m})$ at the image plane, and the RMS radius is $2.5 \mu \mathrm{m}$ on-axis $(x, y=0 \mathrm{~mm})$ and $3.0 \mu \mathrm{m}$ off-axis $(x=0, y=1.5 \mathrm{~mm} ; \mathrm{x}=0, y=-$ $1.5 \mathrm{~mm})$.

In practice, the spatial resolution is again constrained by the pixel size of the sensor and the magnification of the system, resulting in an achievable resolution of $3.5 \mu \mathrm{m}$ (Group 8 Element 4, green box), or $362 \mathrm{lps} / \mathrm{mm}$, at the center of the FOV with full resolution at 11 FPS. A resolution of $6.2 \mu \mathrm{m}$ is achievable with $2 x$ horizontal and vertical binning to run at an acquisition rate of 23 FPS. The magnification is 1.56 . In this 3.5-mm-WD configuration, the full FOV can reach $3.6 \times 2.7 \mathrm{~mm}$, larger than the 1.8-mm-WD counterpart with a smaller magnification achieved.

\section{Imaging place cells in hippocampal CA1}

Studies of the neural representations of space have been prominent ever since the discovery of place cells in the 1970 ' ${ }^{61}$. Many pyramidal neurons within the dorsal hippocampus of rats respond when the animal occupies a particular location within an environment ${ }^{62}$. Neurons with such a spatial response are called "place cells" and it is believed the hippocampus constructs an internal map of an environment (a "cognitive map") which is crucial for spatial navigation and memory ${ }^{17,63}$. Due to the laminar nature of the CA1 pyramidal layer and relative ease of access using implanted optical probes, many experiments on spatial navigation and episodic memories have begun using miniaturized endoscopy in this brain region ${ }^{17,22,64}$. However, most experiments have been restricted to usage in mice, where their smaller size lends to less tissue scattering of light. Development of this MiniLFOV device was focused to overcome the limitations of previous iterations by providing a more powerful and sensitive imaging system for larger animal models. Here we performed $\mathrm{Ca}^{2+}$ imaging in the hippocampal dorsal CA1 region of rats to demonstrate the feasibility and extended capabilities of MiniLFOV recordings in freely behaving larger animal models.

Currently available open-source UCLA Miniscopes support imaging up to around a $1 \mathrm{~mm}^{2} \mathrm{FOV}$, which is sufficient to yield hundreds or thousands of imaged neurons in the mouse hippocampus ${ }^{25}$. However, in animal models that are larger than mice, a larger FOV is often needed to achieve similar cells counts. Furthermore, $\mathrm{Ca}^{2+}$ imaging in larger animals can pose additional difficulties: larger brain sizes can produce greater tissue scattering, and GCaMP expression can be suboptimal using commercially available viral vectors that are often optimized for use in mouse models. A high sensitivity, large FOV miniscope system is needed to ameliorate these problems. 
bioRxiv preprint doi: https://doi.org/10.1101/2021.11.21.469394; this version posted November 22, 2021. The copyright holder for this preprint (which was not certified by peer review) is the author/funder, who has granted bioRxiv a license to display the preprint in perpetuity. It is made available under aCC-BY-NC-ND 4.0 International license.

To record place cell activity from the dorsal CA1 region in rats, $1.2 \mathrm{uL}$ of AAV9-Syn- GCaMP7s was injected just below the pyramidal layer (-3.6 AP, $2.5 \mathrm{ML}, 2.6 \mathrm{DV}$ ) in Long-Evans rats at 3 months old (Charles River). A week later, a custom, $1.8 \mathrm{~mm}$-diameter relay GRIN probe (constructed by stacking two $1.8 \mathrm{~mm}$-diameter GRIN lenses, NA 0.55, \#64-519, Edmund Optics) was surgically implanted above the CA1 region after aspiration of the overlying cortex and corpus callosum (Fig. 3a). Three weeks later, rats were again placed under anesthesia for baseplating. Briefly, the MiniLFOV was mounted into a baseplate with two set screws (4-40 x 1/4"), then positioned above the implanted lenses while the baseplate was cemented in place with bone cement (Fig. 3b). The baseplate body was 3D printed $(20 \mathrm{~mm} \times 20 \mathrm{~mm}$ outer dimensions) with 2 set screws on the side (Fig. 1a, Fig. s3-s4) for rigidly mounting a protective cap when not recording (protecting the GRIN lens and surgical region) and MiniLFOV body during experiments. An overview of installing and removing the MiniLFOV is shown in Figure
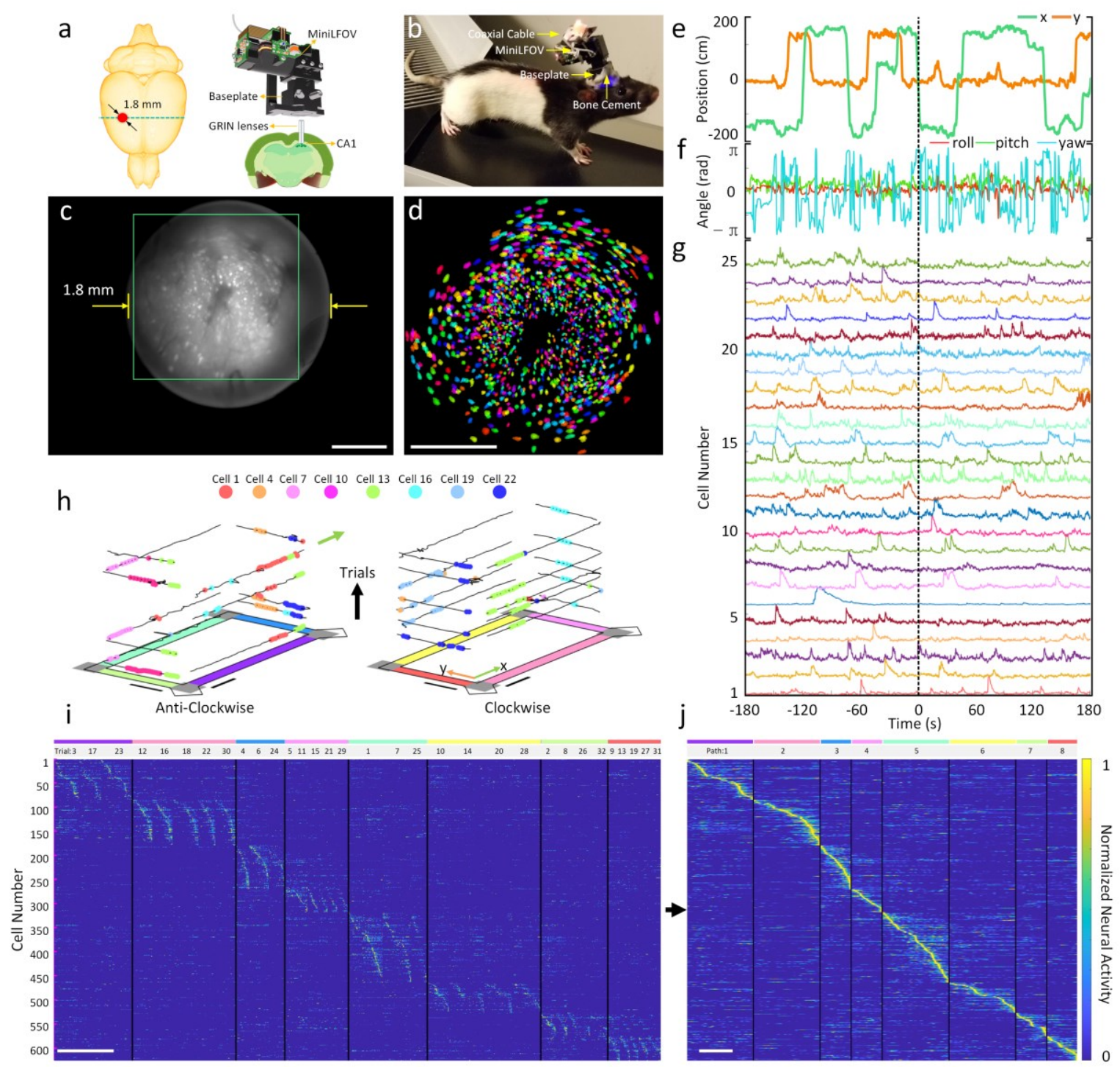

Figure 3. Strategy for imaging Hippcampal dorsal CA1 in freely behaving rats. (a) Schematic of the imaging area in the rat brain. A custom relay GRIN lens is implanted above CA1 to relay fluorescence to the intermediate image plane formed between the top of the GRIN lens and bottom of the MiniLFOV. (b) Rat with MiniLFOV mounted on the skull via the reinforced MiniLFOV baseplate (c) Maximum projection from a 15-minute single recording session after motion correction. Scale bar: $500 \mu \mathrm{m}$. (d) Pixel area outlined in the green box in c showing extracted contours of cells $(\mathrm{N}=1357)$ from CNMF-E analysis via CalmAn, colored randomly. Scale bar: $500 \mu \mathrm{m}$. (e) Example $x$ and $y$ track position as the rat runs in the 2D environment. (f) Example head orientation in terms of the roll, pitch, and yaw Eular angles. (g) Example extracted $\mathrm{Ca}^{2+}$ traces with CalmAn pipeline for 25 cells recorded during the session after low-pass filtering. (h) Deconvolved neural activity of 8 example place cells spatially plotted on top of traversals along the track divided up by trail number and direction. Scale bar: $50 \mathrm{~cm}$. (i) 626 place cells' normalized deconvolved neural activity across all arm traversals sorted by the location of peak spatial activity rate shown in $\mathbf{j}$. Data shown here is speed thresholded $(20 \mathrm{~cm} / \mathrm{s})$ to remove bouts of stationary active along traversals. Scale bar: $10 \mathrm{~s}$. (j) Normalized spatial neural activity rate along all paths for these 626 place cells. Scale bar: $125 \mathrm{~cm}$. 
s4. At around a total mass of 20 grams (which includes the MiniLFOV, baseplate, and cement), rats can easily wear the whole device without any signs of difficulty when running (Fig. 3b). An additional red tracking LED onboard the MiniLFOV's Rigid-Flex PCB is attached on the top of MiniLFOV body and used for tracking the position of rats during experiments.

For the experiment, we imaged $\mathrm{Ca}^{2+}$ dynamics in GCaMP7s-expressing neurons in dorsal CA1 while rats performed a dual choice linear maze task. In total, imaging data from 15 rats across months of recordings were recorded with the MiniLFOV in this task, but we present one session of one rat to demonstrate the effectiveness of the system. Figure 3c shows a maximum projection image from an example 15-minute motion corrected video of CA1 captured by the MiniLFOV. The raw video is $972 \times 1296$ pixels after within-sensor $2 \times 2$ pixel binning and then further cropped to the pixel region containing the relay GRIN lens $(720 \times 720$ pixels). In this example recording, a total number of 1357 cells can be detected and extracted using CNMF-E analysis via the CalmAn pipeline $^{38,39}$ (Fig. 3d). Recordings took place on a rectangular track (2.5 m $\times 1.25 \mathrm{~m}$; Fig. s5), with two reward feeders on two corners of the maze, controlled by a computer running Cheetah software (Neuralynx) feeding position information to custom MATLAB scripts which controlled pellet delivery as the rat ran from one reward zone to the other. Positions on the track (Fig. 3e) and head orientation (Fig. 3f) of the rat were extracted from software synchronized behavioral camera and on-board head orientation sensor respectively (see Methods and Fig. s5). Low-pass filtered $\mathrm{Ca}^{2+}$ traces of 25 example cells recorded in this environment are shown in Figure $3 \mathbf{g}$.

As expected, MiniLFOV imaging of pyramidal neurons in dorsal CA1 during navigation of a rectangular track environment show a significant population of the cells $(626 ; 46.2 \%)$ that are spatially tuned along at least one direction on the 4 arms on the rectangular track. A cell is considered a place cell if it has significantly higher spatial information than chance (spatial information above at least $95 \%$ of 500 resampled randomly shuffled transients for each cell) $)^{65}$. The behavior of the rat was separated into anti-clockwise paths (path 1, 3, 5, and 7) and clockwise paths (path 2, 4, 6, and 8), with each colored differently (Fig. 3h-j). Place cells recorded during the session were significantly modulated by both position and direction of travel and represented all locations along the maze (Fig. 3i-j). An example video showing the position of the rat, head orientation, and place cell activity during behavior is given in Video 2 and Video 3. These data demonstrate how this imaging system can improve experimental efficacy by yielding more cells in larger research models, broadening the horizon of potential hypotheses for researchers. Demonstration of the MiniLFOV's capability in terms of consistently recording activity from the same population of cells across multiple sessions is shown in Figure s6.

\section{Imaging cortex in head-fixed mice}

With single cell resolution across the entire $3.6 \mathrm{~mm} \times 2.7 \mathrm{~mm}$ FOV (3.5-mm-WD configuration), the MiniLFOV is capable of large-scale imaging of cortical ensembles in head-fixed mice, freely moving rats, and larger animals. Here, we demonstrate that MiniLFOV can be used to image with single cell resolution across the entire FOV by imaging the dorsal cortex ${ }^{66}$ in head-fixed mice with the 3.5-mm-WD configuration as shown in Figure 2f, g. A 4 $\mathrm{mm} \times 4 \mathrm{~mm}$ cranial window was used to image neuronal activity in GCaMP6f injected head-fixed mice running on a circular treadmill (22.9-cm diameter). A MiniCAM (Fig. s7a), an open-source behavior camera developed by the UCLA Miniscope group, was used to image, and extract the movement speed of the mouse (Fig. s7b). The MiniLFOV was attached to an articulated ball-head holder mounted on three linear translation stages (XR25C/M, Thorlabs) so that the lateral position and height of the MiniLFOV relative to the cranial window can be adjusted to optimize image quality and cell counts (Fig. s7a, Fig. 4a). Single cell $\mathrm{Ca}^{2+}$ activity could be detected throughout the FOV (Fig. 4a). Motion correction and source extraction using the CalmAn pipeline (as described in the previous section) allowed us to extract putative neurons throughout the FOV. Using deconvolved $\mathrm{Ca}^{2+}$ activity, we classified neurons into one of three categories based on their activity modulation during locomotion. Spatial footprints of the 1,903 identified cells are shown in Figure $\mathbf{4 b}$ distributed across the full FOV. Running speed of the mouse on the circular treadmill was thresholded to produce bouts of "locomotion" and "resting" activity using a threshold of $0.35 \mathrm{~cm} / \mathrm{s}$, with locomotion bouts shown as purple bars under the speed trace (Fig. 4c(top)). Activity dynamics of many neurons throughout the FOV were either positively or negatively modulated during locomotion. We sorted neurons based on their significance of modulation during periods of locomotion compared to rest (Fig 4c). For ease of communication, a cell is classified as a "locomotion cell" if the difference in neural activity rate during locomotion and rest is significantly higher than chance (above at least $95 \%$ of 500 circularly shuffled distributions) and a cell is classified as a "resting cell" if its difference in neural 
activity rate during locomotion and rest is significantly lower than chance (below at least $5 \%$ of 500 circularly shuffled distributions). Many cells could be classified as "locomotion cells" ( $P \geq 0.95 ; \mathrm{N}=434 ; 22.8 \%)$, significantly increasing their transient rate during periods of locomotion or classified as "resting cells" (green; $\mathrm{N}=276 ; 14.5 \%$ ),

significantly suppressing their activity during the same periods. As expected, the mean normalized neural activity of cells classified as locomotion cells clearly shows increased activity during bouts of movement while the mean normalized neural activity of cells classified as resting cells shows the opposite (Fig. 4c(bottom)). Such locomotion related modulation of neuronal activity has been well documented in mouse cortical areas ${ }^{67-69}$. Demonstration of the optical performance of MiniLFOV with single resolution in terms of extracting cells related to locomotion and rest compared to non-single-cell-resolution method is shown in Figure s8. Similar results with a second mouse are shown in Video 4 and Figure s9.
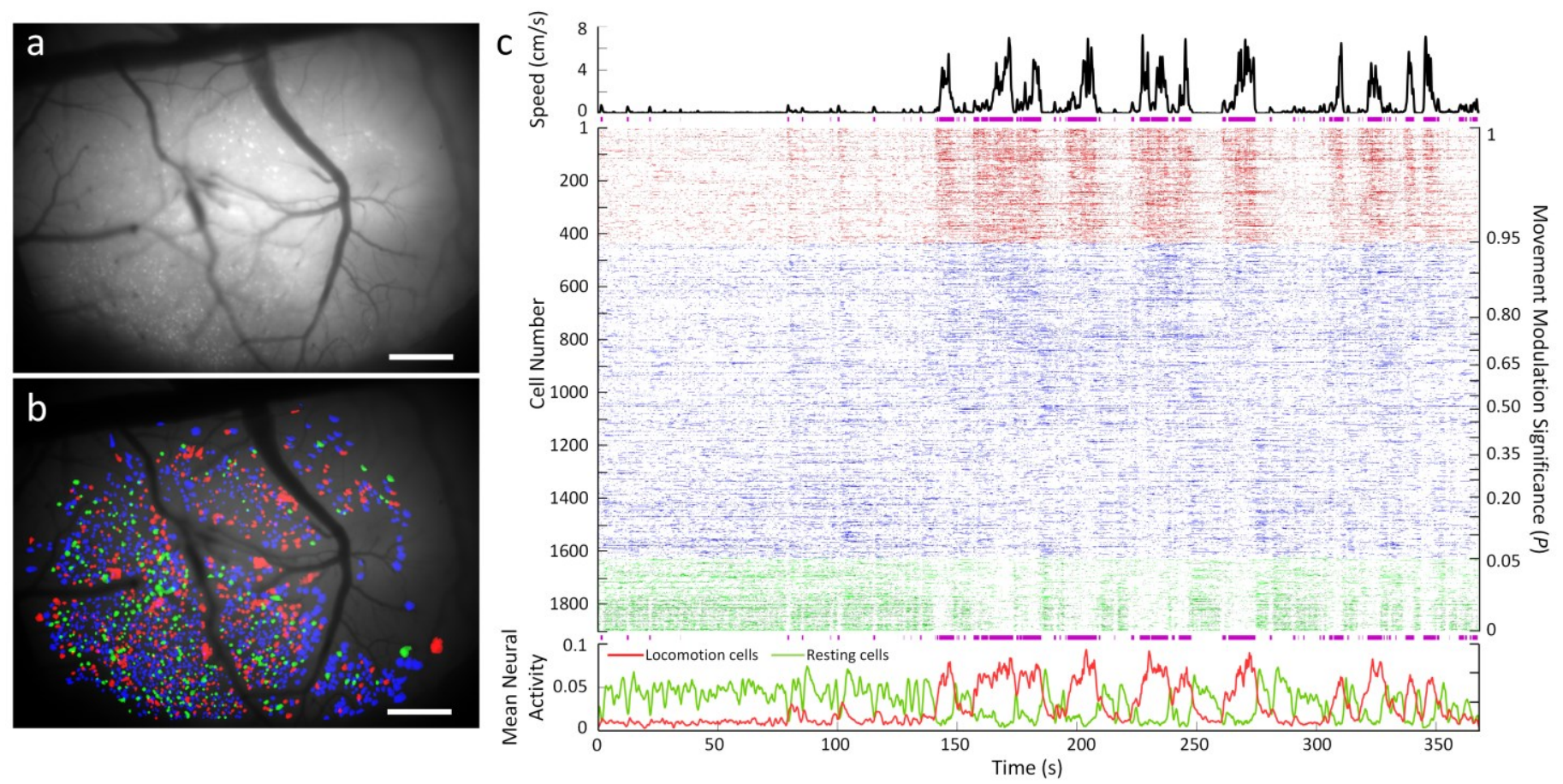

Figure 4. $\mathrm{Ca}^{2+}$ imaging of dorsal cortex through a $4 \mathrm{~mm} \times 4 \mathrm{~mm}$ cranial window in head-fixed mice. (a) Maximum projection from a 7-minute recording session after motion correction. Scale bar: $500 \mu \mathrm{m}$. (b) Contours of extracted cells from CNMF-E analysis via CalmAn (colored based on modulation of neuronal activity during locomotion) overlayed on the minimum projection of the recording session ( $\mathrm{N}=1903$ cells). Scale bar: $500 \mu \mathrm{m}$. (c) Relationship between the circular treadmill motion and extracted deconvolved neural activity. (Top) Circular treadmill speed trace. Purple bars under speed trace denote periods of locomotion. (Middle) Normalized deconvolved neural activity of all extracted neurons. Neurons are sorted based on their significance of activity modulation during periods of locomotion and rest. "Locomotion cells": red, $\mathrm{N}=434,22.8 \%$; "other cells": blue, $\mathrm{N}=1193,62.7 \%$; and "resting cells": green, $\mathrm{N}=276,14.5 \%$. (Bottom) Mean of the normalized deconvolved activity of "locomotion cells" (red) and "resting cells" (green).

\section{Optional Wire-free recording in rats}

The MiniLFOV is capable of recording neural activity without the constraint of being tethered to DAQ hardware mounted off the animal. This is achieved by mounting a novel wire-free DAQ system to the side of the MiniLFOV or on a backpack and connecting it to the MiniLFOV through a single, short $50 \Omega$ coaxial cable carrying power, low bandwidth communication, and high bandwidth imaging data (Fig. 5a, b). Importantly, the wire-free DAQ is compatible with all previous generations of wired open-source UCLA Miniscopes. This wire-free configuration includes the novel wire-free DAQ PCB (Fig. 5c, d) with a microSD card mounted on it for local data storage. For validation of the optional wire-free MiniLFOV, the wire-free DAQ was directly attached to the side of the headmounted MiniLFOV. A single $4 \mathrm{~cm}$ long thin coaxial cable connected the MiniLFOV and wire-free DAQ carrying power and data. A single-cell $400 \mathrm{mAh}$ lithium-polymer battery $(7.5 \mathrm{~g})$ is used to power the DAQ and MiniLFOV, and a 64 GB microSD (Class 10 UHS-I microSD, Kingston) card is used for on-board data and configuration storage (Fig. 5b). Once the wire-free DAQ is powered on, an on-board SAME70 microcontroller (MCU) 
(ATSAME70N21A, Microchip) reads configuration data from the microSD card and then implements that configuration in the MiniLFOV using the $\mathrm{I}^{2} \mathrm{C}$ protocol. Configuration parameters include excitation LED intensity, focus of the EWL, and frame rate, gain, and resolution window of the CMOS image sensor. The wire-free DAQ uses an infrared (IR) remote control receiver to receive digital commands, encoded into a $38 \mathrm{KHz} I R$ carrier frequency, from an IR transmitter to start and stop wire-free recording remotely. This implementation of $I R$ communication allows for one-way, arbitrary, wireless data transfer to the wire-free DAQ and MiniLFOV. Recording starts when the wire-free DAQ receives the IR start code sent from an off-the-shelf IR remote control (KIT-14677, SparkFun) or custom IR remote control transmitter. During recording, data from the attached MiniLFOV is streamed into the memory of the MCU by a direct memory access channel. Each acquired frame is timestamped and then saved onto the SD card using the SD v2.0 interface. The data acquisition rate of the wirefree DAQ is limited by the on-board MCU's parallel capture mode to be at most 8-bit pixel depth at a maximum pixel clock rate of around $24 \mathrm{MHz}$. Additionally, the MCU can maintain a maximum of $\sim 11 \mathrm{MBps}$ write speed to the microSD card, placing additional constrains on the rate of image acquisition and storage of microSD. Due to these constrains, we chose the recording window size to be $608 \times 608$ pixels at $15 \mathrm{FPS}$ (although it can achieve $20 \mathrm{FPS}$ ), corresponding to $1.4 \times 1.4 \mathrm{~mm}^{2} \mathrm{FOV}$ in the object space (relay GRIN lens is $1.8 \mathrm{~mm}$ in diameter).

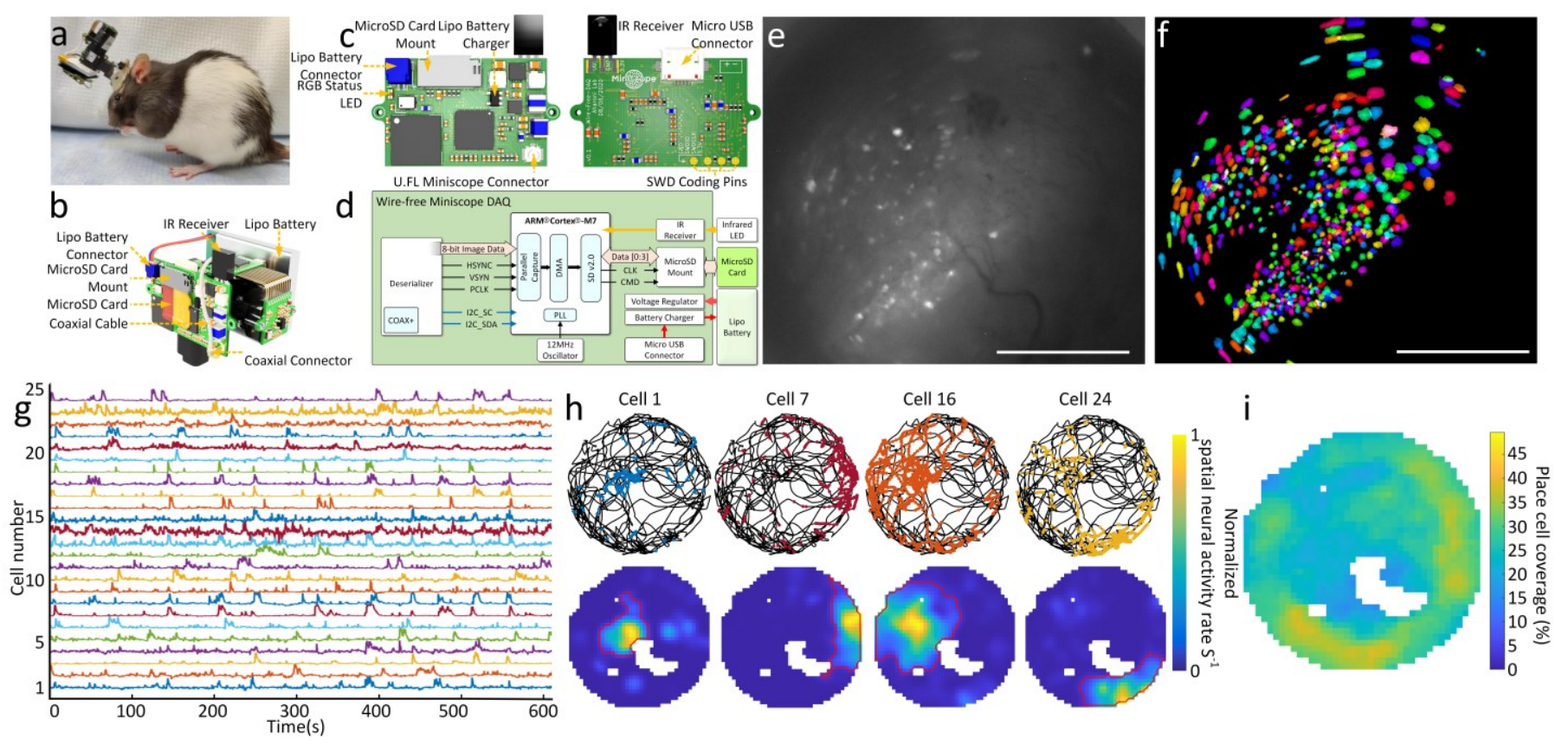

Figure 5. Wire-free imaging of hippocampal CA1 neurons in freely behaving rats in a circular open-field. (a) Photograph of a rat wearing MiniLFOV with wire-free DAQ and single-cell lithium-polymer battery. (b) 3D rendering of the MiniLFOV equiped with wirefree DAQ and single-cell lithium-polymer battery. (c) Top and bottom PCB rendered layouts. (d) Block schematic of wire-free DAQ. (e) Maximum projection from a 14-minute recording session after motion correction. Scale bar: $500 \mu \mathrm{m}$. (f) Contours of extracted cells $(\mathrm{N}=575)$ from CNMF-E analysis via CalmAn, colored randomly. Scale bar: $500 \mu \mathrm{m}$. (g) Low-pass filtered Ca ${ }^{2+}$ transients from 25 example cells from $\mathbf{f}$. (h) Examples of spatially modulated neural activity for 4 place cells during exploration in the open-field. Top: Speed thresholded spatial location of deconvolved neural activity (colored dots) superimposed over rat's trajectory (black line). Bottom: Binned spatial neural activity rate maps for the same example cells. Red contour denotes the spatial outline of a place field based on a greater than $5 \%$ threshold of the binned spatial neural activity rate. (i) Combined open-field place field coverage from all 438 detected place cells $(76 \%)$ showing full coverage of the environment with overall increased coverage near edges.

In the configuration used, around 5.5 MB are written to the SD card per second, allowing a $64 \mathrm{~GB}$ microSD card to support multi-hour recording. The average combined current draw of the wire-free DAQ and MiniLFOV from a single-cell lithium-polymer battery is $460 \mathrm{~mA}$ and therefore a $400 \mathrm{mAh}$ battery can support close to 1 hour of continuous recording. A red, green, blue (RGB) status LED is integrated into the wire-free DAQ for displaying the current device state and visually synchronizing recording with one or multiple behavior cameras (Fig. 5c). Once the wire-free DAQ is powered up, the status LED is illuminated green and then switches to blue once the wire-free DAQ has fully configurated the attached MiniLFOV. Once remotely triggered over IR to start recording, 
the LED turns red and this transition from blue to red can be detected by behavioral cameras and used as a common time point for off-line data synchronization.

The feasibility and capability of the wire-free MiniLFOV configuration are validated with an example rat freely moving in a circular open-field (80-cm diameter) as unrestricted behavior and $\mathrm{Ca}^{2+}$ dynamics in GCaMP7sexpressing neurons in dorsal CA1 are recorded simultaneously. Chocolate flavored sucrose grain pellets (20 mg) were thrown in the enclosed environment every 20 seconds at random locations within the open field, keeping the animal in continuous locomotion, thereby allowing for near complete sampling of the environment ${ }^{70}$. The maximum projection of a motion corrected 14-minute recording session is shown in Figure 5 e with a total of 575 cells extracted using CNMF-E analysis via CalmAn (Fig. 5f). Low-pass filtered $\mathrm{Ca}^{2+}$ traces from 25 example cells are shown in Figure $\mathbf{5 g}$. A significant population of cells $(\mathrm{N}=438 ; 76 \%)$ satisfied our place cell criteria with spatial information above at least $95 \%$ of 500 circularly shuffled sessions. Four example place cells show the rat's trajectory (black lines) and spatial location of deconvolved neural activity (colored dots) (Fig. 5h (top)) showing clear place preference of neural activity. These example cells' spatial neural activity rate maps are shown in Figure $5 \mathrm{~h}$, calculated by dividing the speed-thresholded deconvolved neural activity per bin $(2 \mathrm{~cm} \times 2 \mathrm{~cm})$ by the rat's speed-thresholded occupancy time per bin (Fig. 5h (bottom)). The red outlined contour denotes the thresholded edge of a place field calculated by detecting a $5 \%$ cutoff of the binned spatial neural activity rate surrounding the place field. The combined open-field place field coverage from all 438 detected place cells show full coverage of the circular open-field with overall increased coverage near the edges (Fig, 5i). This optional wire-free configuration enables untethered behavior during neural imaging in larger animals. This has the potential to improve naturalistic behavior by the removing external torque and looping cue generated by tethering while also extending the capabilities of the MiniLFOV, and all other wired UCLA Miniscope systems, to simultaneous multi-animal recordings and experimental designs where tethering is infeasible.

\section{Discussion}

We present a large field of view (FOV) open-source miniature microscope platform aimed at extending the capabilities of microendoscopic imaging approaches in large rodents and non-human primates, such as rats, marmosets, and macaque monkeys. The system can image up to a $3.6 \mathrm{~mm} \times 2.7 \mathrm{~mm}$ FOV at 23 FPS with single cell resolution, has an electrically adjustable working distance of up to $3.5 \mathrm{~mm} \pm 150 \mu \mathrm{m}$ adopting an onboard EWL, incorporates an absolute head-orientation sensor, and weighs under 14 grams. The MiniLFOV achieves a 20-fold increase in sensitivity compared to the previous generation Miniscope V3, and a 2-fold increase in sensitivity compared to the current generation mouse Miniscope V4. The MiniLFOV includes a modular objective lens configuration, enabling multiple imaging approaches such as deep brain imaging using implanted optical probes and cortical imaging through cranial windows. The MiniLFOV can be easily attached to and removed from a chronically implanted baseplate on the head of the animal that has been designed to provide rigid mechanical support for stable recording in freely behaving animals larger than a mouse. The baseplate mounting mechanism also provides an accurate and repeatable mounting to image the same neural population and track individual neurons across recording sessions (Figure s6).

The MiniLFOV has been validated in freely behaving rats by imaging GCaMP7s expressing neurons in the dorsal CA1 layer of the hippocampus and is one of first demonstrations of large-scale recordings of place cells in freely behaving rats. Previous recordings in our lab of freely behaving rats using original Miniscope V3 designed for mice had been severely limiting, typically yielding around 100 cells or fewer per recording ${ }^{59}$. With the presented MiniLFOV, we can now achieve simultaneous recordings of over 1,000 neurons in freely behaving rats and place cell counts of over 600 cells in a single rat during a single 15-minute session (Fig. 3). When imaging across the entire FOV of the MiniLFOV, close to 2,000 active neurons could be imaged across the dorsal cortex in headfixed mice (Fig. 4, Video 4). This platform greatly extends $\mathrm{Ca}^{2+}$ imaging capabilities in freely behaving larger animal models and opens up new avenues of research that were previously limited by low simultaneous cell counts. Additionally, the entire open-source UCLA Miniscope platform has also been extended for wire-free operation. This has the potential to reduce behavior interference (such as cable tangling and unintentional looming cues) as well as enable recordings from multiple animals socially interacting ${ }^{71}$ and animals exploring large environments. Performance and capabilities of three configurations of the MiniLFOV, i.e., 1.8-mm-WD, 3.5$\mathrm{mm}-\mathrm{WD}$, and wire-free, are listed in Table 1. Furthermore, with independently controlled dual-LED excitation, 
the MiniLFOV can support future two-color excitation configurations by changing one of the LEDs as well as changing filters and dichroic mirror. With a mass of only 13.9 grams, two MiniLFOVs (28 grams) can be mounted onto the skull of a rat for multi-region imaging. More than two MiniLFOVs can be mounted simultaneously on even larger animals with the open-source Miniscope DAQ software being able to support at least 5 MiniLFOV data streams. In summary, the MiniLFOV broadens the application of $\mathrm{Ca}^{2+}$ imaging to larger animal models than previously feasible, allowing for multiple cortical areas can be imaged simultaneously and correlations and interactions between distant neuronal populations ${ }^{33,42,72}$ can be investigated to understand, for example, the neural activity that supports sensory coding and motor output ${ }^{73}$. Moreover, it provides an open-source, accessible, and cost-effective option for neuroscientists seeking to adopt $\mathrm{Ca}^{2+}$ imaging into their labs.

\section{References}

1. Chen, T. W. et al. Ultrasensitive fluorescent proteins for imaging neuronal activity. Nature 499, (2013).

2. Denk, W., Strickler, J. H. \& Webb, W. W. Two-photon laser scanning fluorescence microscopy. Science (80-. ). (1990) doi:10.1126/science.2321027.

3. Denk, W. \& Svoboda, K. Photon upmanship: Why multiphoton imaging is more than a gimmick. Neuron (1997) doi:10.1016/S0896-6273(00)81237-4.

4. Lu, R. et al. Rapid mesoscale volumetric imaging of neural activity with synaptic resolution. Nat. Methods (2020) doi:10.1038/s41592-020-0760-9.

5. Ozbay, B. N. et al. Three dimensional two-photon brain imaging in freely moving mice using a miniature fiber coupled microscope with active axial-scanning. Sci. Rep. (2018) doi:10.1038/s41598-018-26326-3.

6. Li, Z. et al. Fast widefield imaging of neuronal structure and function with optical sectioning in vivo. Sci. Adv. 6, (2020).

7. Wei Chen, Ryan G. Natan, Yuhan Yang, Shih-Wei Chou, Qinrong Zhang, E. Y. I. \& N. J. In vivo volumetric imaging of calcium and glutamate activity at synapses with high spatiotemporal resolution. Nat. Commun. 12, (2021).

8. Wang, T. et al. Three-photon imaging of mouse brain structure and function through the intact skull. Nat. Methods 15, (2018).

9. Seiriki, K. et al. High-Speed and Scalable Whole-Brain Imaging in Rodents and Primates. Neuron 94, (2017).

10. Men, Y. et al. Exosome reporter mice reveal the involvement of exosomes in mediating neuron to astroglia communication in the CNS. Nat. Commun. 10, (2019).

11. Keller, P. J., Ahrens, M. B. \& Freeman, J. Light-sheet imaging for systems neuroscience. Nature Methods (2014) doi:10.1038/nmeth.3214.

12. Voleti, V. et al. Real-time volumetric microscopy of in vivo dynamics and large-scale samples with SCAPE 2.0. Nat. Methods (2019) doi:10.1038/s41592-019-0579-4.

13. Fan, J. et al. Video-rate imaging of biological dynamics at centimetre scale and micrometre resolution. Nat. Photonics 13, (2019).

14. Robinson, N. T. M. et al. Targeted Activation of Hippocampal Place Cells Drives Memory-Guided Spatial Behavior. Cell (2020) doi:10.1016/j.cell.2020.09.061.

15. Dombeck, D. A., Harvey, C. D., Tian, L., Looger, L. L. \& Tank, D. W. Functional imaging of hippocampal place cells at cellular resolution during virtual navigation. Nat. Neurosci. (2010) doi:10.1038/nn.2648.

16. Ghosh, K. K. et al. Miniaturized integration of a fluorescence microscope. Nat. Methods 8, (2011).

17. Ziv, Y. et al. Long-term dynamics of CA1 hippocampal place codes. Nat. Neurosci. (2013) doi:10.1038/nn.3329.

18. Sawinski, J. et al. Visually evoked activity in cortical cells imaged in freely moving animals. Proc. Natl. 
bioRxiv preprint doi: https://doi.org/10.1101/2021.11.21.469394; this version posted November 22, 2021. The copyright holder for this preprint (which was not certified by peer review) is the author/funder, who has granted bioRxiv a license to display the preprint in perpetuity. It is made available under aCC-BY-NC-ND 4.0 International license.

Acad. Sci. U. S. A. 106, (2009).

19. Zong, W. et al. Fast high-resolution miniature two-photon microscopy for brain imaging in freely behaving mice. Nat. Methods (2017) doi:10.1038/nmeth.4305.

20. Klioutchnikov, A. et al. Three-photon head-mounted microscope for imaging deep cortical layers in freely moving rats. Nat. Methods (2020) doi:10.1038/s41592-020-0817-9.

21. Zong, W. et al. Miniature two-photon microscopy for enlarged field-of-view, multi-plane and long-term brain imaging. Nat. Methods (2021) doi:10.1038/s41592-020-01024-z.

22. Cai, D. J. et al. A shared neural ensemble links distinct contextual memories encoded close in time. Nature (2016) doi:10.1038/nature17955.

23. Aharoni, D., Khakh, B. S., Silva, A. J. \& Golshani, P. All the light that we can see: a new era in miniaturized microscopy. Nature Methods (2019) doi:10.1038/s41592-018-0266-x.

24. Aharoni, D. \& Hoogland, T. M. Circuit investigations with open-source miniaturized microscopes: Past, present and future. Frontiers in Cellular Neuroscience (2019) doi:10.3389/fncel.2019.00141.

25. Shuman, T. et al. Breakdown of spatial coding and interneuron synchronization in epileptic mice. Nat. Neurosci. (2020) doi:10.1038/s41593-019-0559-0.

26. Liberti, W. A., Perkins, L. N., Leman, D. P. \& Gardner, T. J. An open source, wireless capable miniature microscope system. J. Neural Eng. (2017) doi:10.1088/1741-2552/aa6806.

27. Barbera, G. et al. Spatially Compact Neural Clusters in the Dorsal Striatum Encode Locomotion Relevant Information. Neuron (2016) doi:10.1016/j.neuron.2016.08.037.

28. Liang, B. et al. Distinct and Dynamic ON and OFF Neural Ensembles in the Prefrontal Cortex Code Social Exploration. Neuron (2018) doi:10.1016/j.neuron.2018.08.043.

29. de Groot, A. et al. Ninscope, a versatile miniscope for multi-region circuit investigations. Elife (2020) doi:10.7554/eLife.49987.

30. Jacob, A. D. et al. A compact head-mounted endoscope for in vivo calcium imaging in freely-behaving mice. bioRxiv (2018) doi:10.1101/252205.

31. Skocek, O. et al. High-speed volumetric imaging of neuronal activity in freely moving rodents. Nat. Methods (2018) doi:10.1038/s41592-018-0008-0.

32. Yanny, K. et al. Miniscope3D: optimized single-shot miniature 3D fluorescence microscopy. Light Sci. Appl. 9, (2020).

33. Gonzalez, W. G., Zhang, H., Harutyunyan, A. \& Lois, C. Persistence of neuronal representations through time and damage in the hippocampus. Science (80-. ). 365, (2019).

34. lii, L. Large-scale cellular-resolution imaging of neural activity in freely behaving mice. bioRxiv (2021).

35. Scherrer, J. R., Lynch, G. F., Zhang, J. J. \& Fee, M. S. A Novel Optical Design Enabling Lightweight and Large Field-Of-View Head-Mounted Microscopes. bioRxiv 2021.09.03.458947 (2021).

36. Juneau, J. et al. MiniFAST: A sensitive and fast miniaturized microscope for in vivo neural recording. bioRxiv (2020).

37. Giovannucci, A. et al. CalmAn an open source tool for scalable calcium imaging data analysis. Elife (2019) doi:10.7554/eLife.38173.

38. Zhou, P. et al. Efficient and accurate extraction of in vivo calcium signals from microendoscopic video data. Elife (2018) doi:10.7554/eLife.28728.

39. Friedrich, J., Zhou, P. \& Paninski, L. Fast online deconvolution of calcium imaging data. PLoS Comput. Biol. (2017) doi:10.1371/journal.pcbi.1005423.

40. Dong, Z., Mau, W., Feng, Y. (Susie) \& Pennington, Z. T. Minian: An open-source miniscope analysis 
bioRxiv preprint doi: https://doi.org/10.1101/2021.11.21.469394; this version posted November 22, 2021. The copyright holder for this preprint (which was not certified by peer review) is the author/funder, who has granted bioRxiv a license to display the preprint in perpetuity. It is made available under aCC-BY-NC-ND 4.0 International license.

pipeline. bioRxiv 6, (2021).

41. Senarathna, J. et al. A miniature multi-contrast microscope for functional imaging in freely behaving animals. Nat. Commun. 10, (2019).

42. Rynes, M. L. et al. Miniaturized head-mounted microscope for whole-cortex mesoscale imaging in freely behaving mice. Nat. Methods (2021) doi:10.1038/s41592-021-01104-8.

43. Barbera, G., Liang, B., Zhang, L., Li, Y. \& Lin, D. T. A wireless miniScope for deep brain imaging in freely moving mice. J. Neurosci. Methods (2019) doi:10.1016/j.jneumeth.2019.05.008.

44. Yartsev, M. M. Space bats: Multidimensional spatial representation in the bat. Science (80-. ). (2013) doi:10.1126/science.1245809.

45. Yartsev, M. M. \& Ulanovsky, N. Representation of three-dimensional space in the hippocampus of flying bats. Science (80-. ). (2013) doi:10.1126/science.1235338.

46. Bollimunta, A. et al. Head-mounted microendoscopic calcium imaging in dorsal premotor cortex of behaving rhesus macaque. Cell Rep. (2021) doi:10.1016/j.celrep.2021.109239.

47. Geva-Sagiv, M., Las, L., Yovel, Y. \& Ulanovsky, N. Spatial cognition in bats and rats: From sensory acquisition to multiscale maps and navigation. Nature Reviews Neuroscience vol. 16 (2015).

48. Dotson, N. M. \& Yartsev, M. M. Nonlocal spatiotemporal representation in the hippocampus of freely flying bats. Science (80-. ). 373, (2021).

49. Geva-Sagiv, M., Romani, S., Las, L. \& Ulanovsky, N. Hippocampal global remapping for different sensory modalities in flying bats. Nat. Neurosci. 19, (2016).

50. Angelaki, D. E. et al. A gravity-based three-dimensional compass in the mouse brain. Nat. Commun. (2020) doi:10.1038/s41467-020-15566-5.

51. Sargolini, F. et al. Conjunctive representation of position, direction, and velocity in entorhinal cortex. Science (80-. ). (2006) doi:10.1126/science.1125572.

52. Gerlei, K. et al. Grid cells are modulated by local head direction. Nat. Commun. (2020) doi:10.1038/s41467-020-17500-1.

53. Solstad, T., Boccara, C. N., Kropff, E., Moser, M. B. \& Moser, E. I. Representation of geometric borders in the entorhinal cortex. Science (80-. ). (2008) doi:10.1126/science.1166466.

54. Ellenbroek, B. \& Youn, J. Rodent models in neuroscience research: Is it a rat race? DMM Dis. Model. Mech. 9, (2016).

55. Harvey, C. D., Coen, P. \& Tank, D. W. Choice-specific sequences in parietal cortex during a virtualnavigation decision task. Nature 484, (2012).

56. Gauthier, J. L. \& Tank, D. W. A Dedicated Population for Reward Coding in the Hippocampus. Neuron 99, (2018).

57. Mau, W. et al. The Same Hippocampal CA1 Population Simultaneously Codes Temporal Information over Multiple Timescales. Curr. Biol. 28, (2018).

58. Rubin, A. et al. Revealing neural correlates of behavior without behavioral measurements. Nat. Commun. 10, (2019).

59. Hart, E. E., Blair, G. J., O’Dell, T. J., Blair, H. T. \& Izquierdo, A. Chemogenetic modulation and singlephoton calcium imaging in anterior cingulate cortex reveal a mechanism for effort-based decisions. $J$. Neurosci. 40, (2020).

60. Kane, G. A., Lopes, G., Saunders, J. L., Mathis, A. \& Mathis, M. W. Real-time, low-latency closed-loop feedback using markerless posture tracking. Elife 9, (2020).

61. O'Keefe, J. \& Dostrovsky, J. The hippocampus as a spatial map. Preliminary evidence from unit activity 
in the freely-moving rat. Brain Res. (1971) doi:10.1016/0006-8993(71)90358-1.

62. O'Keefe, J. Place units in the hippocampus of the freely moving rat. Exp. Neurol. (1976) doi:10.1016/0014-4886(76)90055-8.

63. O’Keefe, J. \& Nadel, L. Hippocampus as cognitive map. Behav. Brain Sci. (1979).

64. Mau, W., Hasselmo, M. E. \& Cai, D. J. The brain in motion: How ensemble fluidity drives memoryupdating and flexibility. Elife 9, (2020).

65. Skaggs WE, McNaughton BL, Gothard KM, M. E. An Information-Theoretic Approach to Deciphering the Hippocampal Code. Proc. IEEE (1993).

66. Watson, C., Paxinos, G. \& Puelles, L. The Mouse Nervous System. The Mouse Nervous System (2012). doi:10.1016/C2009-0-00185-8.

67. Niell, C. M. \& Stryker, M. P. Modulation of Visual Responses by Behavioral State in Mouse Visual Cortex. Neuron 65, (2010).

68. Mineault, P. J., Tring, E., Trachtenberg, J. T. \& Ringach, D. L. Enhanced spatial resolution during locomotion and heightened attention in mouse primary visual cortex. J. Neurosci. 36, (2016).

69. Pakan, J. M. P. et al. Behavioral-state modulation of inhibition is context-dependent and cell type specific in mouse visual cortex. Elife 5, (2016).

70. Guger, C. et al. Real-time position reconstruction with hippocampal place cells. Front. Neurosci. (2011) doi:10.3389/fnins.2011.00085.

71. Kingsbury, L. et al. Correlated Neural Activity and Encoding of Behavior across Brains of Socially Interacting Animals. Cell 178, (2019).

72. Scott, B. B. et al. Imaging Cortical Dynamics in GCaMP Transgenic Rats with a Head-Mounted Widefield Macroscope. Neuron 100, (2018).

73. Stirman, J. N., Smith, I. T., Kudenov, M. W. \& Smith, S. L. Wide field-of-view, multi-region, two-photon imaging of neuronal activity in the mammalian brain. Nat. Biotechnol. (2016) doi:10.1038/nbt.3594.

74. Frank, A. C. et al. Hotspots of dendritic spine turnover facilitate clustered spine addition and learning and memory. Nat. Commun. 9, (2018).

75. Ravassard, P. et al. Multisensory control of hippocampal spatiotemporal selectivity. Science (80-. ). 340, (2013).

76. Bjerknes, T. L., Dagslott, N. C., Moser, E. I. \& Moser, M. B. Path integration in place cells of developing rats. Proc. Natl. Acad. Sci. U. S. A. 115, (2018).

77. Sheintuch, L. et al. Tracking the Same Neurons across Multiple Days in Ca2+ Imaging Data. Cell Rep. 21, (2017). 


\section{Methods}

MiniLFOV design, manufacturing, and assembly. Design. The optical system of the MiniLFOV was designed using Zemax OpticsStudio. The goal of the optical system is to produce a miniature microscope with large FOV and high NA, in which the desired NA is set to be 0.25 in the objective space and FOV to be a minimum of $3 \mathrm{~mm}$. Lenses are chosen to achieve the optical performance while maintaining a compactable and lightweight size. The distance between lenses is optimized to achieve single cell resolution across the entire FOV with minimal field curvature. Three achromatic lenses $L 1$ (d=6.25 mm, focal length=60 mm, \#45-345, Edmund Optics), L2-1 ( $d=9 \mathrm{~mm}$, focal length=12 mm, \#49-656, Edmund Optics), and L3-1 (d=9 mm, focal length=27 mm, \#45-092, Edmund Optics) are used to build the 1.8-mm-WD objective module with a $3.5 \mathrm{~mm}$ tall spacer placed between L2-1 and L3-1. L1 (d=6.25 mm, focal length=60 mm, \#45-345, Edmund Optics), L2-2 (d=9 mm, focal length=18 $\mathrm{mm}$, \#49-657, Edmund Optics), and L3-2 (d=9 mm, focal length=36 mm, \#45264, Edmund Optics) are chosen to build the 3.5-mm-WD objective module with a $2 \mathrm{~mm}$ tall spacer placed between L1 and L2-2 (Fig. 2d, f). One aspherical lens ( $d=12.5 \mathrm{~mm}$, focal length=14 mm, \#49-658, Edmund Optics) and a concave lens ( $d=12 \mathrm{~mm}$, focal length=-48 mm, \#45-019, Edmund Optics) with a lens holder are placed into the emission module body to form the tube lens of the MiniLFOV. The EWL (Corning Arctic 58N, Varioptics/Corning) is placed into the sensor module body (Fig. 1d, Fig. s1) for electronic focus adjustment. The MiniLFOV module bodies (objective module body, emission module body, sensor module body), filter cover, baseplate, and protective cap are designed in Autodesk Fusion 360 (educational license) and printed with black resin (FLGPBK04, Formlabs) by an SLA 3D printer (Form 3, Formlabs). Three filter slots are designed on the emission module body for mounting a custom diced excitation filter (4 $4 \times 1.1 \mathrm{~mm}$, ET470/40x, Chroma), a dichroic filter (14 $\times 10 \times 1 \mathrm{~mm}$, T500spxr, Chroma) and an emission filter $(10 \times 10 \times 1 \mathrm{~mm}, \mathrm{ET} 525 / 50 \mathrm{~m}$, Chroma). All the optical components can be easily assembled without the need for any epoxy or optical glue. A list of the optical components used is given in Table 2. The circuit schematic and Rigid-Flex PCB layout are designed using KiCad, a free software suite for electronic design automation (EDA). The PCB is divided up into 4 rigid sub-circuits which include an excitation LED circuit, an electrowetting lens tuning and head orientation circuit, a CMOS image sensor circuit, and a power-over-coax and serializer circuit. The modularity of the PCB design enables quick modification or redesign of individual subcircuits without the need for modifying the entire PCB layout. The four sub-circuits are connected by a doublesided embedded flex printed circuit (Fig. 1a, b). The assembled objective module is attached to the emission module body with five 18-8 Stainless Steel Socket Head Screws (92196A052, McMaster-Carr). The emission module, sensor module, cover, and Rigid-Flex PCB are fastened together with M1 thread-forming screws (96817a704, McMaster-Carr) (see Fig. s1). The open-source behavior camera (MiniCAM) for behavior tracking is developed by UCLA Miniscope group (https://github.com/Aharoni-Lab/MiniCAM) and compatible with the open-source UCLA Miniscope DAQ hardware and software. The MiniCAM consists of an M12 optical lens mount, a custom printed circuit board housing a CMOS image senor and supporting electronics, LED illumination ring with 16 red LEDs, and a 3D printed case. The brightness of the LEDs can be adjusted through software for the optimal illumination in dark environments. The MiniCAM runs at around 50 FPS with $1024 \times 768$ resolution and saves the behavioral data in AVI file format using an MJPG compression video codec directly supported within the UCLA Miniscope software.

Wiring. A single flexible coaxial cable ( $50 \Omega$, CW2040-3650SR, Cooner Wire) is used for power, communication, and image data transmission relying on a passive power-over-coax filter and a seriailzer/deserializer pair ( $\mathrm{TI}$ DS90UB913A/DS90UB914A) for combining and separating DC power, low-speed bi-directional communication, and high-speed uni-directional imaging data. This design minimizes the impact of cabling on animal behavior, supporting coaxial cables down to $0.3 \mathrm{~mm}$ in diameter (Molex 100065-0023) and low-torque, commercial (FL-6C-MICRO, Dragonfly) and DIY commutators. The DAQ hardware and software are based on the UCLA Miniscope project's previous work (http://miniscope.org/index.php/Main Page), with updated firmware (https://github.com/Aharoni-Lab/Miniscope-DAQ-Cypress-firmware) and software (https://github.com/AharoniLab/Miniscope-DAQ-QT-Software) to enable video streaming and controlling of the MiniLFOV. This updated software enables excitation LED brightness adjustment, focus adjustment by $E W L$, real-time $\Delta F / F$ and fluorescent trace visualization, frame rate selection, gain adjustment, and supports real-time pose estimation using DeepLabCut-Live ${ }^{60}$ through embedded Python. A list of the hardware and software used is given in Table 2. 
Animals. All experimental protocols were approved by the Chancellor's Animal Research Committee of the University of California, Los Angeles, in accordance with the US National Institutes of Health (NIH) guidelines.

Surgical implantation. Rat hippocampal imaging. 3-month-old Long-Evans rats (Charles River) underwent two survival surgeries prior to behavior training to record fluorescent $\mathrm{Ca}^{2+}$ activity from hippocampal CA1 cells. During the first surgery, rats were anesthetized with $5 \%$ isoflurane at $2.5 \mathrm{~L} / \mathrm{min}$ of oxygen, then maintained at $2-2.5 \%$ isoflurane while a craniotomy was made above the dorsal hippocampus. Next, $1.2 \mathrm{uL}$ of AAV9-Syn-GCamp7s (AddGene) was injected just below the pyramidal layer (-3.6 AP, 2.5 ML, 2.6 DV) via a 10 uL Nanofil syringe (World Precision Instruments) mounted in a Quintessential Stereotaxic Injector (Stoelting) controlled by a Motorized Lab Standard Stereotax (Harvard Apparatus). Left or right hemisphere was balanced across all animals. One week later, the rat was again induced under anesthesia and 4 skull screws were implanted to provide stable mounting for the GRIN lens implant and MiniLFOV baseplate. The craniotomy was reopened to a diameter of $1.8 \mathrm{~mm}$, and cortical tissue and corpus callosal fibers above the hippocampus were aspirated away using blunted 27 and 30 gauge needles. Following this aspiration, and assuring no bleeding persisted in the craniotomy, a $1.8 \mathrm{~mm}$-diameter GRIN lens (\#64-519, Edmund Optics) was implanted over the hippocampus and cemented in place with methacrylate bone cement (Simplex-P, Stryker Orthopaedics). The dorsal surface of the skull and the bone screws were cemented with the GRIN lens to ensure stability of the implant, while the surface of the lens was left exposed. Two to three weeks later, rats were again placed under anesthesia to cement a 3D printed baseplate above the lens. First a second GRIN lens was optically glued (Norland Optical Adhesive 68, Edmund Optics) to the surface of the implanted lens and cured with UV light. The pitch of each GRIN lens was approximately 0.25 , so combining 2 in series provided roughly a 0.5 pitch. This half pitch provides translation of the image at the bottom surface of the lenses to the top while maintaining the focal point below the lens, effectively becoming a relay GRIN lens. This relay implant enables access to tissue deep below the skull surface. The MiniLFOV was placed securely in the baseplate and then mounted to the stereotax to visualize the $\mathrm{Ca}^{2+}$ fluorescence and tissue. The baseplate was then cemented in place above the relay lenses at the proper focal plane, the MiniLFOV was removed from the baseplate, and cement allowed to cure. Once rats had been baseplated, they were placed on food restriction to reach a goal weight of $85 \%$ ad lib weight and then began behavioral training.

Rat histology. At the end of the experiment, rats were anesthetized with isoflurane, intraperitoneally injected with $1 \mathrm{~mL}$ of pentobarbital, then transcardially perfused with $100 \mathrm{~mL}$ of $0.01 \mathrm{M}$ PBS followed by $200 \mathrm{~mL}$ of $4 \%$ paraformaldehyde in $0.01 \mathrm{M}$ PBS to fix the brain tissue. Brains were sectioned at $40 \mu \mathrm{m}$ thickness on a cryostat (Leica), mounted on slides, then imaged on a confocal microscope (Zeiss) to confirm GFP expression and GRIN lens placement.

Cranial window imaging on head fixed mice. Adult C57BL/6N Tac (3-5 months old) male mice were singly housed on a $12 \mathrm{~h}$ light/dark cycle. Mice were bilaterally microinjected with $500 \mathrm{~nL}$ of AAV1.Syn.GCaMP6f.WPRE.SV40 virus (purchased from Addgene 100837) at $20-120 \mathrm{~nL} / \mathrm{min}$ into the dorsal cortex using the stereotactic coordinates: -1.7 and $-2.3 \mathrm{~mm}$ posterior to bregma, $0.5 \mathrm{~mm}$ lateral to midline and $-0.8 \mathrm{~mm}$ ventral to skull surface. Mice underwent window implantation surgeries as previously described ${ }^{74}$. Briefly, a square region of skull $\sim 4 \mathrm{~mm}$ in width was marked using stereotactic coordinates (center at bregma $-2.2 \mathrm{~mm} \mathrm{AP}$ ). The skull was thinned using a dental drill and removed. After cleaning the surgical site with saline, a custom cut sterilized coverslip (square, $4 \times 4 \mathrm{~mm}$ ) was placed on the dural surface and fastened with adhesive and dental acrylics to expose a square window of approximately $3.5 \mathrm{~mm}$ spanning the midline. Three weeks later, a small 3D printed baseplate was cemented onto the animal's head atop the previously placed dental cement. In the second mouse, an aluminum bar with two threaded holes was attached to stabilize the mice during imaging sessions. Following baseplating or attachment of headbar, mice underwent handling ( 3 days) and habituation (3 days) to acclimate them to the treadmill and head-fixation.

Rats Behavioral Training. $\mathrm{Ca}^{2+}$ imaging in freely behaving rats. After baseplating, rats were given $15-$ minute sessions every 48 hours to perform a linear alternation task on a rectangular linear maze where one arm $(2.5 \mathrm{~m})$ directly connects the reward locations, while the other 3 arms in total are indirect and, combined, twice as long $(5 \mathrm{~m})$. These arms form a $2.5 \mathrm{~m} \times 1.25 \mathrm{~m}$ rectangle with the reward locations at the corners of one long side, elevated 1 meter off the ground. The paths are $10-\mathrm{cm}$ wide spanned by small metal bars along the entirety of 
the short and long paths. The reward zones are coated wood, $14-\mathrm{cm}$ wide, $20-\mathrm{cm}$ long, at a 45-degree angle with the two paths. The entire maze has a short 1-cm wall to provide a safe graspable ledge in case the rat loses its footing. The outer perimeter of the short path has a slightly taller wall angled 45 degrees away from the path to protect the wiring connected to the short path bars. 20-mg chocolate sucrose pellets are delivered through a metal tube at the end of the reward zone via an automated hopper. Rewards are delivered at the opposite reward location from their previous reward as the rat passes through the center of either path. Both paths yield delivery of 2 pellets, and there is no time out period after delivery. The rat must enter the most recent reward delivery location before the opposite side will be rewarded, forcing an alternation behavior. The rats are allowed to choose either path, and since they are equally rewarded, they demonstrate a preference for the short path $(2.5 \mathrm{~m}$ arm) over the long path (the other 3 arms) after only a few sessions. Rats are given 15-minute recording sessions every 48 hours to minimize potential photobleaching of the $\mathrm{Ca}^{2+}$ indicator. Once a rat demonstrated a consistent short path preference and sufficient running behavior (>2 short paths per minute and a 2:1 short:long ratio in the first 10 minutes for two consecutive sessions) we introduced a manipulation in the subsequent session. Effects of these manipulations are omitted in this article, and we only evaluate period of consistent behavioral performance in absence of induced learning.

Wire-free imaging on freely behaving rats. For testing the functionality of the wire-free configuration, the experiment was conducted by putting the rat into a circular open-field $(80-\mathrm{cm}$ diameter) to record the unconstrained exploration of the environment and the neural activity simultaneously. An additional red LED is attached onto the MiniLFOV body for the behavior camera to track its position. Animals were food-deprived up to $85 \%$ of their original weight after which recordings commenced. Sucrose grain pellets $(20 \mathrm{mg})$ were thrown in the enclosed environment every 20 seconds at random locations within the open field, keeping the animal in continuous locomotion, thereby allowing close to complete sampling of the environment ${ }^{70}$.

Data analysis. Behavior video analysis. For experiments involving freely behaving rats, videos of behaviors were captured in AVI format using an overhead camera (30 FPS, Logitech C270). The position of the rat is extracted by tracking the position of the red LED attached to the top of the MiniLFOV recorded by a behavioral camera. The pixel location of the LED is found by detecting the highest pixel value region within the gray-scale behavior recording frames, a correction of the optical aberration of the camera lens is applied, and then the pixel value location is converted to real-world coordinates. For the head-fixed experiment in mice, a MiniCAM (Fig. s7a) was used for recording movement speed of the mice. The movement of the mice was extracted from the recorded motion of a circular treadmill $(22.9-\mathrm{cm}$ diameter) marked with $1-\mathrm{cm}$ wide alternating dark and white bars on the surface (Fig. s7b).

Head orientation analysis. The quaternion values $\left(q_{w}, q_{x}, q_{y}, q_{z}\right)$, read from the on-board head orientation sensor (BNO055, Bosch) following each frame acquisition, were saved in CSV file format during recording. This data was converted to Euler angles (roll, pitch, yaw) offline for further analysis, with the matrix used below:

$$
\begin{gathered}
R=\left[\begin{array}{lll}
r_{11} & r_{12} & r_{13} \\
r_{21} & r_{22} & r_{23} \\
r_{31} & r_{32} & r_{33}
\end{array}\right]=\left[\begin{array}{ccc}
1-2 q_{y}^{2}-2 q_{z}^{2} & 2 q_{x} q_{y}+2 q_{w} q_{z} & 2 q_{x} q_{z}-2 q_{w} q_{y} \\
2 q_{x} q_{y}-2 q_{w} q_{z} & 1-2 q_{x}^{2}-2 q_{z}^{2} & 2 q_{y} q_{z}+2 q_{w} q_{x} \\
2 q_{x} q_{z}+2 q_{w} q_{y} & 2 q_{y} q_{z}-2 q_{w} q_{x} & 1-2 q_{x}^{2}-2 q_{y}^{2}
\end{array}\right] \\
\text { roll }=\arctan \left(\frac{r_{23}}{r_{33}}\right), \text { pitch }=\arctan \left(\frac{\sqrt{r_{11}{ }^{2}+r_{12}}}{-r_{13}}\right), \text { and yaw }=\arctan \left(\frac{\sin (\text { roll }) \times r_{31}-\cos (\operatorname{roll}) \times r_{21}}{\cos (\text { roll }) \times r_{22}-\sin (\text { roll }) \times r_{32}}\right)
\end{gathered}
$$

Further correction of the Euler angles depends on the axis orientation of the head-orientation sensor and the mounting orientation of the MiniLFOV during experiments.

MiniLFOV data preprocessing. The imaging data from the CMOS image sensor is saved in AVI file format using an uncompressed (GREY) video codec directly supported within the Miniscope DAQ software, and all the recorded videos for each animal in each session were concatenated into one single video file and cropped to the ROI pixel region using custom Python 3 scripts before further processing.

Wired experiment on rats in rectangular linear maze. Image stacks of $\mathrm{Ca}^{2+}$ dynamics (972 $\times 1296$ pixels) were cropped to the pixel region containing the relay lens stack $(720 \times 720$ pixels). We next used the CalmAn Python 
processing pipeline to perform non-rigid motion correction followed by image source extraction using constrained nonnegative matrix factorization for endoscopic recordings (CNMF-E) ${ }^{38,39}$ to identify and extract the spatial contours and fluorescent $\mathrm{Ca}^{2+}$ activity of individual neurons. Fast non-negative deconvolution OASIS (https://github.com/zhoupc/OASIS matlab) was used to deconvolve the slow time course inherent in the GCaMP fluorophore ${ }^{39}$ to estimate underlying neural activity. The resulting deconvolved $\mathrm{Ca}^{2+}$ activity can be interpreted as the sum of neural activity within each frame scaled by an unknown constant, and we refer to this measure as the 'temporal neural activity' of a cell. Because the temporal neural activity of each cell is scaled by an unknown number that varies across cells, we normalized each cell's temporal neural activity. Spatial neural activity rates were calculated path by path (Path 1 to 8 ) using 2-cm-wide spatial bins and a speed threshold of greater than $20 \mathrm{~cm} / \mathrm{s}$. Temporal neural activity and occupancy of the animal were spatially binned and then smoothed using a Gaussian kernel with $\sigma=5 \mathrm{~cm}$. Then the binned neural activity was divided by the binned occupancy to calculate the spatial neural activity rate of each cell.

The information content of the spatial neural activity rate map (in bits) ${ }^{75}$ of a single neuron was defined as:

$$
I=\sum_{i=1}^{N} p_{i} \frac{\lambda_{i}}{\bar{\lambda}} \log _{2} \frac{\lambda_{i}}{\bar{\lambda}}, p_{i}=\frac{t_{i}}{\sum_{i=1}^{N} t_{i}}, \bar{\lambda}=\sum_{i=1}^{N} p_{i} \lambda_{i}
$$

where $t_{i}$ represents the occupancy time spent in the i-th bin of total $N$ bins and $\lambda_{i}$ represents the neural activity rate in the i-th bin. $\bar{\lambda}$ is the mean neural activity rate. The significance of the spatial information was calculated using two circular shuffling procedures. For each shuffling procedure, the random permutations were generated by frame-shifting the speed-thresholded sequence of temporal neural activity for each trail (total 32 trials) by a random interval between 0 and the number of frames for each trial respectively with frame number out of range being circularly wrapped to the beginning ${ }^{76}$. Then random permutations were generated by frame-shifting the entire sequence of temporal neural activity along the rat's 8 paths by a random interval between 0 and the number of frames, with frame number out of range being circularly wrapped to the beginning. This procedure was repeated 500 times with random shifts to determine a significance measure for the spatial activity rate map of each neuron. The significance measure is the percentile of the true information content value within the distribution of the information content values from the 500 randomly shuffled sessions. Cells information content significantly above chance $(P \geq 0.95)$ based on the circularly shuffled distribution are labelled place cells.

Head-fixed experiment imaging dorsal cortex in mice. As described earlier, $\mathrm{Ca}^{2+}$ recordings were processed as to yield the spatial contours of neurons and their deconvolved $\mathrm{Ca}^{2+}$ activity. We next calculated the neural activity rate for each cell during periods of locomotion $\left(r_{\mathrm{m}}\right)$ and rest $\left(r_{\mathrm{s}}\right)$ by summing the neural activity in either state divided by the amount of time spent in locomotion or rest, respectively. The threshold adopted on the running speed to define a period as "locomotion" and "rest" is set to be $0.35 \mathrm{~cm} / \mathrm{s}$ for Mouse 1 and $0.23 \mathrm{~cm} / \mathrm{s}$ for Mouse 2 as these best matched experimenter's report of circular treadmill at rest vs in motion for each mouse. The difference in neural activity rate during locomotion and rest $\left(r_{m}-r_{s}\right)$ was then used for calculating significant locomotion modulation using a circular shuffling procedure. Random permutations were generated by frameshifting the entire sequence of temporal neural activity along the recording by a random interval between the start and the end of recording, with the end being circularly wrapped to the beginning. This procedure was repeated 500 times to determine a significance measure for each neuron. The significance measure is the percentile of the true value within the distribution of the values from the 500 randomly shuffled sessions. A value above chance $(P \geq 0.95)$ based on the circularly shuffled distribution indicates the cells have significantly increased transient rate during periods of locomotion and were classified as "locomotion cells". Likewise, a value below chance $(P \leq 0.05)$ indicated suppressed neural activity during the same period and these cells were classified as "resting cells". The cells with significance between these ranges $(0.05<P<0.95)$ are classified as "other cells". Then the sum of neural activity of "locomotion cells" was divided by the number of "locomotion cells" to calculate the mean normalized neural activity of "locomotion cells" and the sum of neural activity of "resting cells" was divided by the number of "resting cells" to calculate the mean normalized neural activity of "resting cells".

Wire-free imaging on freely behaving rats. Wire-free MiniLFOV data was extracted from microSD cards and saved as uncompressed 8 bit AVI video files using custom Python code for processing and analysis. Ca ${ }^{2+}$ recordings were processed as described earlier to yield the spatial contours of neurons and their deconvolved 
$\mathrm{Ca}^{2+}$ activity. Spatial neural activity rates were calculated using $2 \mathrm{~cm} \times 2 \mathrm{~cm}$ spatial bins and a speed threshold of greater than $5 \mathrm{~cm} / \mathrm{s}$. Temporal neural activity and occupancy of the animal were spatially binned and then smoothed using a Gaussian kernel with $\sigma=3 \mathrm{~cm}$. A minimum occupancy threshold was set to be $100 \mathrm{~ms}$ where spatial bins that did not meet this threshold were excluded from all subsequent analysis. The binned neural activity was divided by the binned occupancy to calculate the spatial neural activity rate of each cell. Information content of the spatial neural activity rate map was calculated with the same equation as described in the wired experiment on rats in the rectangular track and the significance of the spatial information content was calculated using a circular shuffling procedure. Random permutations were generated by time-shifting the entire sequence of positions along the rat's path by a random interval between $10 \mathrm{~s}$ and the total recording minus $10 \mathrm{~s}$, with the time out of the range being wrapped to the beginning ${ }^{76}$. The 10-seconds at the beginning and the end of the recoding are occluded to remove the period of putting the rats into and out of the open-field. This procedure was repeated 500 times to determine a significance measure for the spatial activity rate map of each neuron. The significance measure is the percentile of the true information content value within the distribution of the information content values from the 500 randomly shuffled sessions. Cells with information content significantly above chance $(P \geq 0.95)$ based on the circularly shuffled distribution are labelled place cells. For cells defined as place cells, their place fields are defined as the region that 1) contains 5 adjacent bins with binned neural activity rate above $95 \%$ of that of all randomly shuffled binned neural activity rates and 2) extends to all connected bins with a binned neural activity rate of at least $5 \%$ of the place field's peak binned neural activity rat. This approach allows for a robust detection and spatial region definition for place cells with single as well as multiple place fields. The place field spatial region is shown as red contours in Figure $\mathbf{5 h}$.

Matching cells across sessions. Contours computed from CalmAn were normalized by their own maximum value then thresholded at 0.5 to generate compact contours for all cells. Artifact contours detected on the edge of the GRIN lens were manually removed then each session was manually aligned using the contours and vasculature simultaneously using custom MATLAB scripts. In addition to linear translations, it was sometimes necessary to radially scale this data as well to account for slight differences induced by camera placement and different focal positions from the electrowetting lens. For all rats, we aligned and matched 3 consecutive recording sessions (48 hours apart) where running performance was stable. Contour alignment was performed using CellReg to register cells across all sessions of interest based on their centroid distances and spatial contour correlations ${ }^{77}$. Distributions of centroid distances and spatial correlation for all cell pairs within $24 \mu \mathrm{m}$ were computed, yielding a two-dimensional distribution that can be modeled and given a probability threshold to match cell pairs. Cell pairs with a probability $>0.5$ for both the centroid and correlation distributions were matched (low centroid distance and high spatial correlation), which is expected when recording the same cell.

\section{Data availability}

The experimental data that support the findings of this study are available from $\mathrm{H}$. Tad Blair(blairlab@gmail.com), Alcino J. Silva(silvaa@mednet.ucla.edu), and Daniel Aharoni (DAharoni@mednet.ucla.edu) upon reasonable request.

\section{Code availability}

Data analysis scripts are available on reasonable request from H. Tad Blair(blairlab@gmail.com), and Daniel Aharoni (DAharoni@mednet.ucla.edu).

\section{ACKNOWLEGMENTS}

Changliang Guo was supported by the National Institute of Mental Health (1UF1NS107668).

Garrett Blair was supported by NeuroNex grant, National Science Foundation (award \# 1707408).

Megha Sehgal was supported by R01 MH113071, Adelson Adelson Medical Research Foundation and DBI1707408. 
Federico N.Sangiuliano Jimka was supported by NeuroNex grant, National Science Foundation (award \# 1707408).

Arash Bellafard was supported by NIH Grant 1R01NS116589-01.

\section{AUTHOR CONTRIBUTIONS}

Daniel Aharoni, Changliang Guo, H. Tad Blair, Michele A. Basso, Peyman Golshani, and Alcino J. Silva conceived the study design.

Daniel Aharoni, and Changliang Guo developed the MiniLFOV.

Federico N.Sangiuliano Jimka contributed to dual-LED circuit design.

Garrett Blair performed the surgeries in rats.

Garrett Blair, and Changliang Guo conducted the rat experiments.

Megha Seghal performed the cranial window surgeries in mice.

Megha Sehgal, Changliang Guo, and Garrett Blair conducted the head-fixed experiments on mice.

Arash Bellafard contributed to testing MiniLFOV on head-fixed mice.

Changliang Guo and Garrett Blair performed data analysis.

Changliang Guo, Garrett Blair, Megha Sehgal, and Daniel Aharoni prepared the manuscript and figures.

\section{COMPETING FINANCIAL INTERESTS}

The authors declare no competing interests. 
bioRxiv preprint doi: https://doi.org/10.1101/2021.11.21.469394; this version posted November 22, 2021. The copyright holder for this preprint (which was not certified by peer review) is the author/funder, who has granted bioRxiv a license to display the preprint in perpetuity. It is made available under aCC-BY-NC-ND 4.0 International license.

\section{Supplemental Information}

Miniscope-LFOV: A large field of view, single cell resolution, miniature microscope for wired and wire-free imaging of neural dynamics in freely behaving animals 
(a)

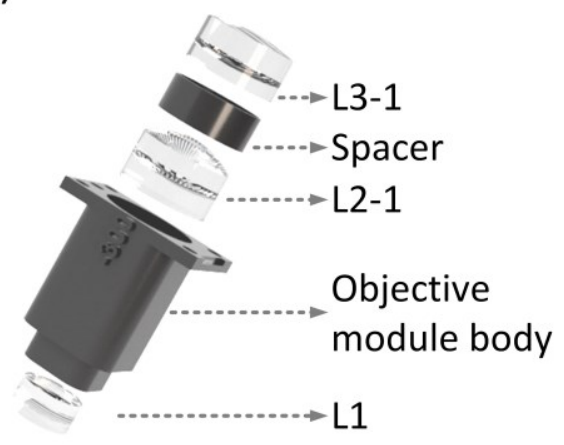

(c)

Coaxial Connector
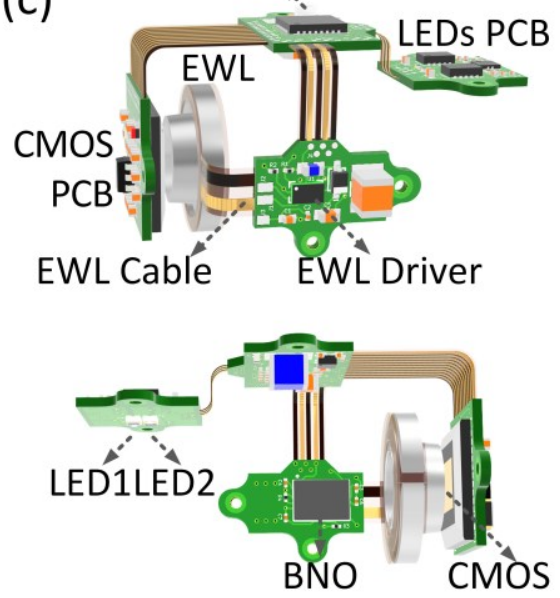

(b)

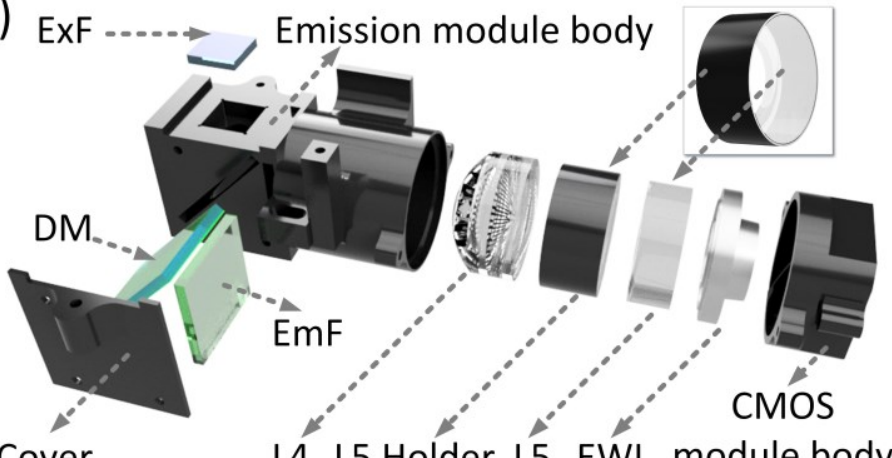

Cover
L4 L5 Holder L5 EWL module body

(d)
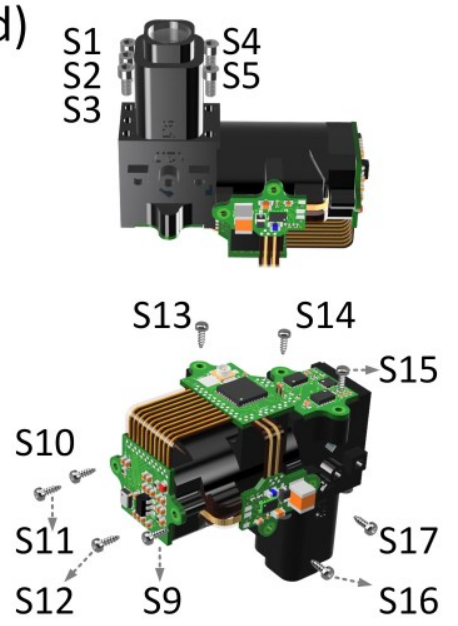
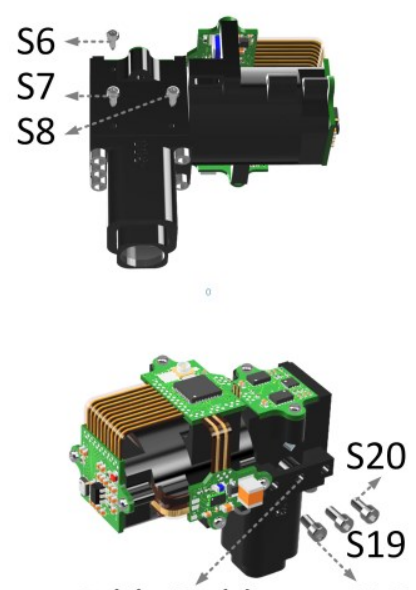

Cable Holder S18

Figure s1. Mechanical and optical assembly of MiniLFOV. The MiniLFOV consists of three main modules, objective module (details in a), emission module (details in $\mathbf{b}$ ), sensor module, and a custom Rigid-Flex printed circuit board (PCB) (details in c), and they are screwed together with two types of screws (details in d). Objective module (1.8-mm WD) contains three achromatic lenses (L1, L2-1, and L3-1) with a spacer (3.5-mm tall) placed between L2-1 and L3-1, with a 3D printed objective module body to hold the components. 3.5-mm-WD objective module contains three achromatic lenses (L1, L2-2, and L3-2) with a spacer (2-mm tall) placed between L1 and L2-2. Emission module contains filters (excitation filter "ExF", dichroic mirror "DM", and emission filter "EmF"), and optics lenses (achromatic L4 and plane-concave lens L5), with a 3D printed emission module body to hold the filters and lenses. Sensor module holds the EWL with a $3 \mathrm{D}$ printed CMOS module body. The circuit module consists of 4 sub-circuits, an excitation LED circuit, an electrowetting lens tuning and head orientation circuit, a CMOS image sensor circuit for collecting $\mathrm{Ca}^{2+}$ imaging data, and a power-over-coax and serializer circuit (Fig. 1a, b) for supporting coaxial cable power and data transmission. Two LEDs are housed on the LED circuit board driven by two LED drivers with $\mathrm{I}^{2} \mathrm{C}$ digital potentiometer for brightness adjustment. Electrowetting lens tuning and head orientation circuit consists of EWL driver, with EWL cable holding the EWL, to adjust the focus of the EWL and the absolute-orientation chip for collecting head orientation data. 5M CMOS sensor (MT9P031) is housed on the CMOS image sensor circuit for $\mathrm{Ca}^{2+}$ fluorescence image capturing. A serializer chip on the power-overcoax and serializer circuit serializes the imaging data and sends it over a coaxial connector to communicate with a custom MiniscopeDAQ system. The objective module, emission module, sensor module and the circuits module are assembled by 12 (S6-S17) M1 thread-forming screws (96817a704, McMaster-Carr), and 8 (S1-S5, S18-S20) 18-8 stainless steel socket head screws (0-80 Thread Size, 92196A052, McMaster-Carr). 
(a)

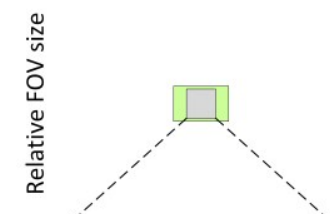

(b)

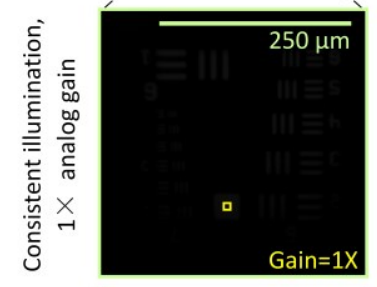

(c)

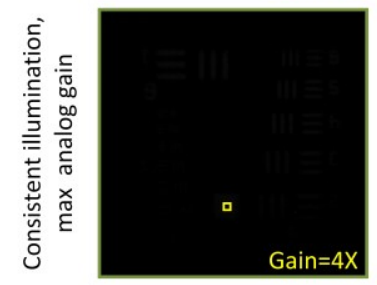

(d)

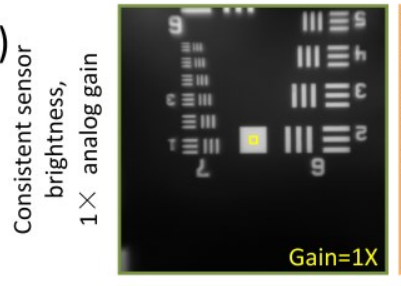

(e)

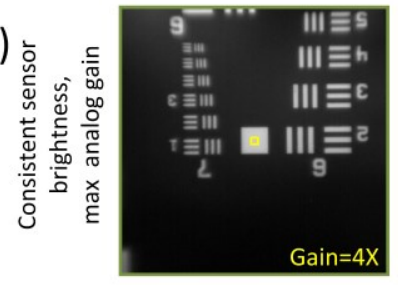

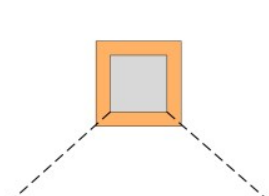
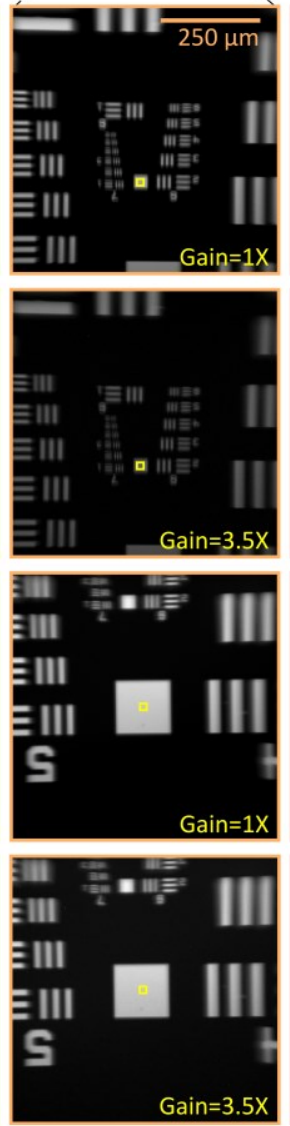
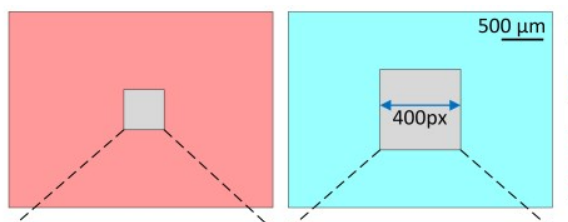

$\square$ Miniscope V3 $(6 \mu \mathrm{m}, 752 \times 480)$
$\square$ Miniscope V4 $(4.8 \mu \mathrm{m}, 600 \times 600)$
$\square$ MiniLFOV_full $(2.2 \mu \mathrm{m}, 2592 \times 1944)$
$\square$ MiniLFOV_bin $2 \times(4.4 \mu \mathrm{m}, 1296 \times 972)$
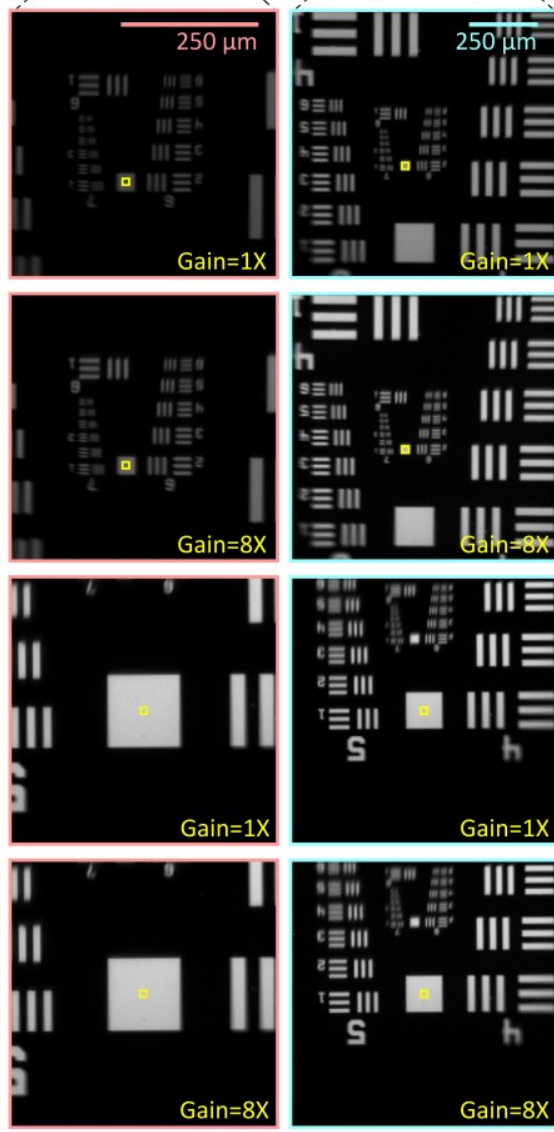
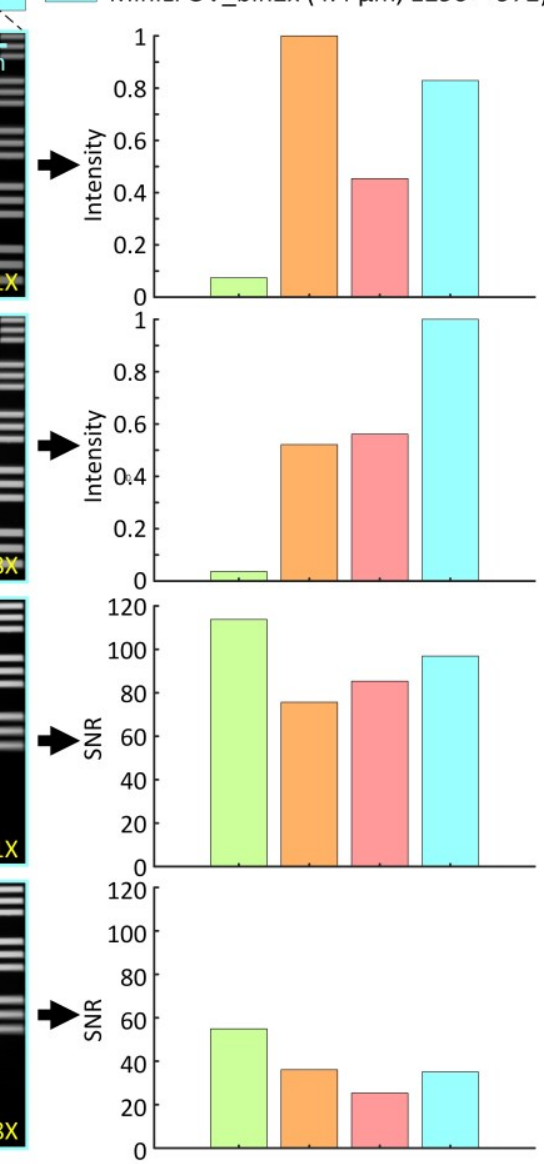

Figure s2. Sensitivity and signal noise ratio (SNR) comparison of Miniscope V3, Miniscope V4, MiniLFOV full resolution (MiniLFOV_full), and MiniLFOV after $2 \times 2$ pixel binning (MiniLFOV_bin2x). A light source was placed under a Negative USAF $1951 \mathrm{Hi}$-Resolution Target (\#55-622, Edmund Optics) with a thin diffuser placed between them to diffuse the light. Then a Miniscope V3, Miniscope V4, and MiniLFOV are attached to a $3 \mathrm{D}$ translation stage and placed above the target. The lateral position and height of the Miniscopes were adjusted to optimize image quality for each imaging session. A 30-second video was recorded with each Miniscope in cases of consistent illumination ( $1 \mathrm{x}$, and max analog gain), and consistent sensor brightness ( $1 \mathrm{x}$, and max analog gain). The sensitivity comparison of the Miniscopes was done by illuminating the back side of the Negative USAF $1951 \mathrm{Hi}$-Resolution Target with the light source remaining at a constant intensity. Sensor sensitivity was calculated as the mean pixel value within the $10 \times 10$ pixel box shown in yellow. The SNR comparison is was done by adjusting the light source intensity to achieve equivalent pixel brightness (8bit gray scale value $=\sim 200$ ), across all Miniscopes in the chosen area on the sensors. Sensor SNR was calculated as the mean pixel value divided by the standard deviation of all pixels within the $10 \times 10$ pixel box shown in yellow. A $400 \times 400$ pixel mean image from each 30-second recording is shown. (a) Relative FOV size of Miniscope V3 (light green box, $650 \mu \mathrm{m} \times 420 \mu \mathrm{m}$ ), Miniscope V4 (orange box, 1-mm diameter), MiniLFOV (salmon, and cyan, $3.1 \mathrm{~mm} \times 2.3$ $\mathrm{mm}$ ), and chosen $400 \times 400$ pixel area (gray box) in each case. Legend shows pixel size and full sensor resolution. (b) Sensitivity of Miniscopes with unity analog gain set for each. MiniLFOV is less sensitive than Miniscope V4 $(83 \%)$. (c) Sensitivity of Miniscopes with maximum analog gain set for each. Higher sensitivity is achieved with MiniLFOV than Miniscope in this case (192\%). (d) SNR of Miniscopes with unity analog gain set for each. (e) SNR of Miniscopes with maximum analog gain set for each. 

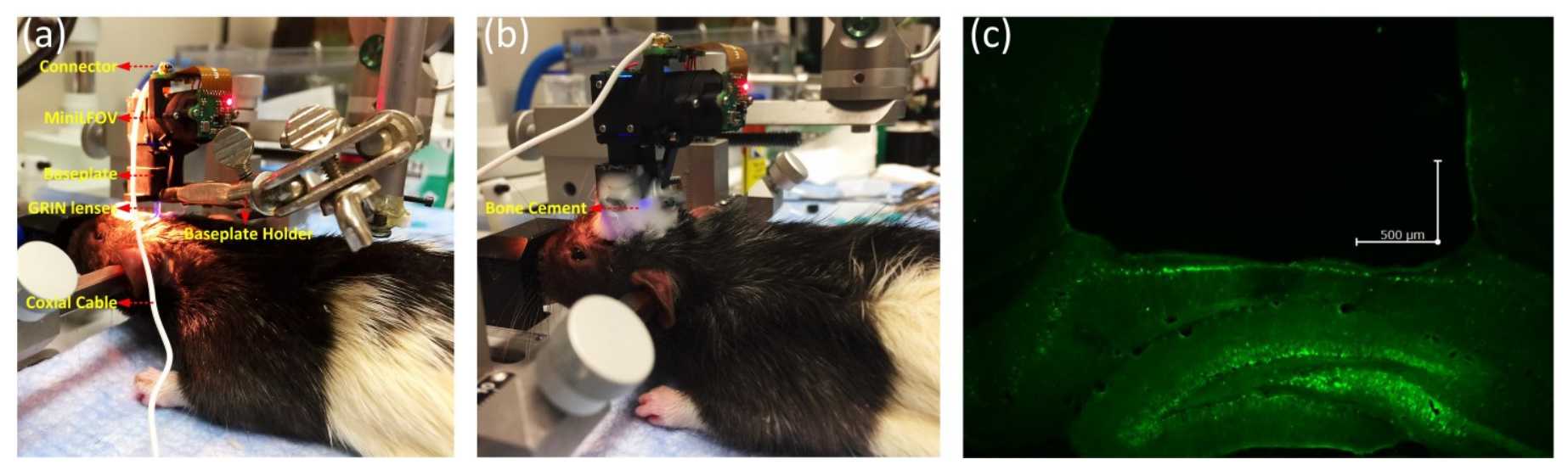

Figure s3. MiniLFOV baseplating on the rat. (a) A second $\sim 0.25$-pitch GRIN lens is affixed atop of the first $\sim 0.25$-pitch GRIN lens (implanted during earlier surgery), to construct a relay optics setup ( $\sim 0.5$ pitch). Then the MiniLFOV baseplate is held by a stereotaxic clamp. The MiniLFOV, attached to the baseplate with two screws, is positioned above the second GRIN lens on an anesthetized ear-barred rat. The distance from the MiniLFOV to GRIN lens is adjusted to focus on the hippocampal CA1 region exhibiting the most active and in-focus neurons. (b) The MiniLFOV baseplate is then affixed to the animal via bone cement mixture. After baseplating, a protective cap is used to cover and protect the GRIN lens from dust and scratches. (c) Histological image of a brain slice using a confocal microscope (Zeiss) sectioned at 40- $\mu$ m thickness on a cryostat (Leica) to confirm GFP and GRIN lens placement. Picture shows GFP expression in the dorsal hippocampus of the rat, approximately 2.6 $\mathrm{mm}$ below the skull surface; scale bar: $500 \mu \mathrm{m}$. 
bioRxiv preprint doi: https://doi.org/10.1101/2021.11.21.469394; this version posted November 22, 2021. The copyright holder for this preprint (which was not certified by peer review) is the author/funder, who has granted bioRxiv a license to display the preprint in perpetuity. It is made available under aCC-BY-NC-ND 4.0 International license.
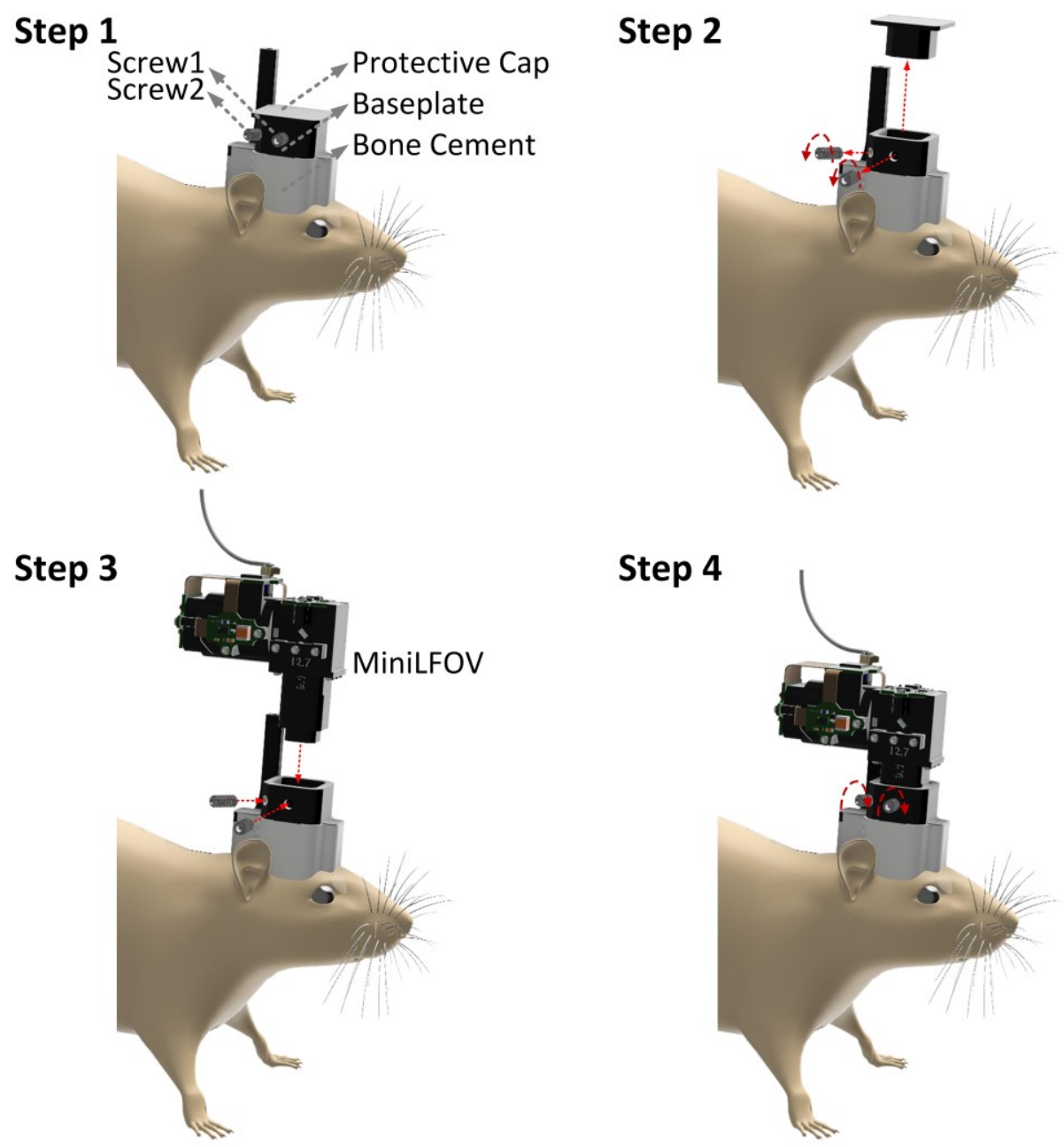

Figure s4. Mounting the MiniLFOV on the rat's head. (Step 1) While the animal is in its home cage, a protective cap is used in the baseplate and secured with two set screws (4-40 x 1/4") to protect the GRIN lens from dust and scratches. At the start of the experiment, the protective cap is removed (Step 2) and the MiniLFOV is inserted into the baseplate and secured with two set screws (Step 3-4). At the end of the imaging session, the MiniLFOV is again replaced by the protective cap. 
bioRxiv preprint doi: https://doi.org/10.1101/2021.11.21.469394; this version posted November 22, 2021. The copyright holder for this preprint (which was not certified by peer review) is the author/funder, who has granted bioRxiv a license to display the preprint in perpetuity. It is made available under aCC-BY-NC-ND 4.0 International license.
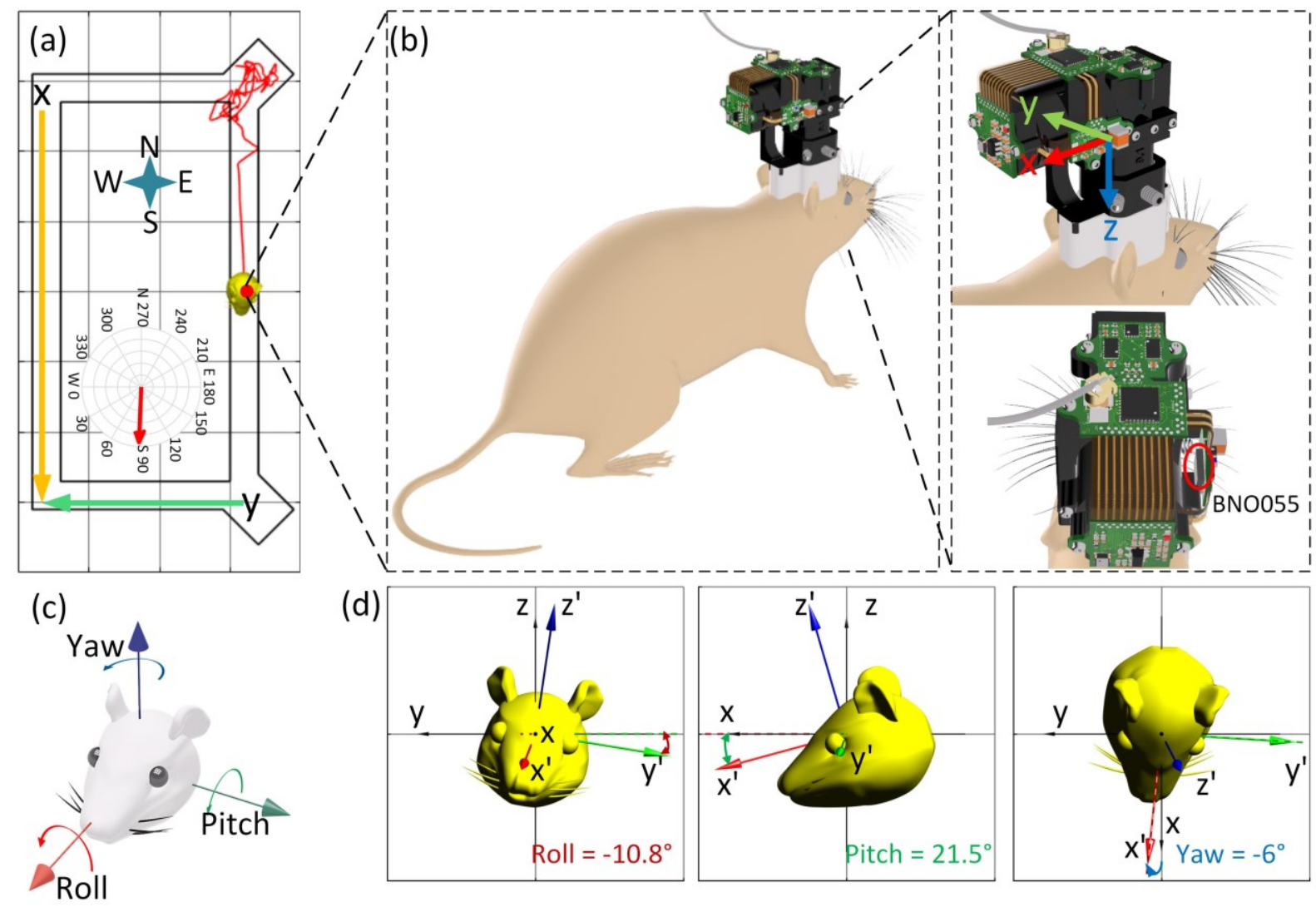

(d)
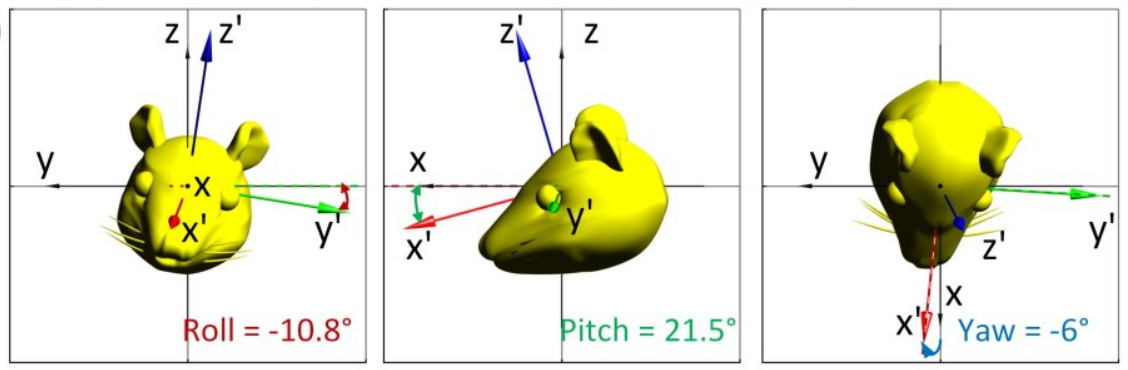

Figure s5. Illustration of the head orientation data (roll, pitch, yaw) from head-orientation sensor (BNO055). (a) A rat with a MiniLFOV mounted on its head running on a rectangular track. (b) 3D image showing the coordinate of BNO055 configured and its orientation. The axis orientation of the BNO055 can be reconfigured to the new reference axis before experiment. (c) Illustration of roll, pitch, and yaw in terms of direction and rotation. (d) Zoomed-in figures shows the example roll $\left(-10.8^{\circ}\right)$, pitch $\left(21.5^{\circ}\right)$, and yaw $\left(-6^{\circ}\right)$ extracted from the recorded quaternion value $\left(q_{w}, q_{x}, q_{y}, q_{z}\right)$, when the rat is passing $\mathrm{x}=0$, running from north to the south on the track. 
(a)
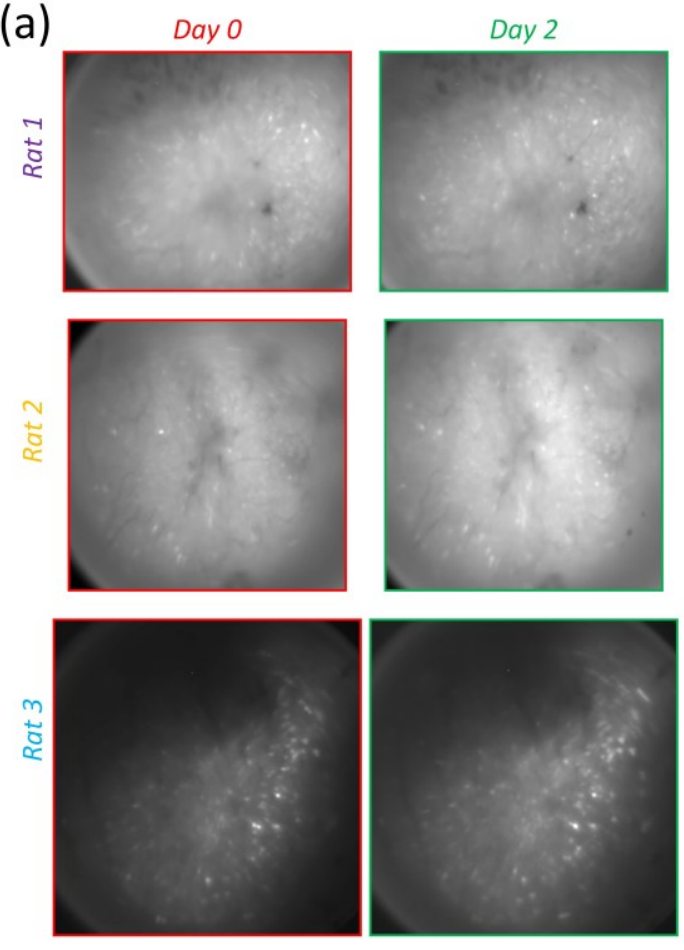
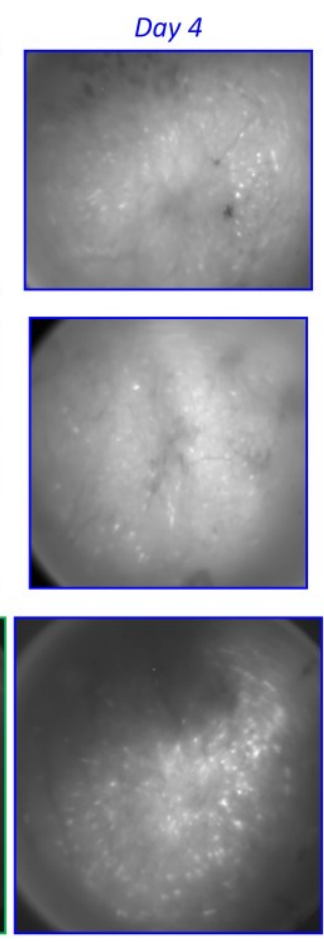
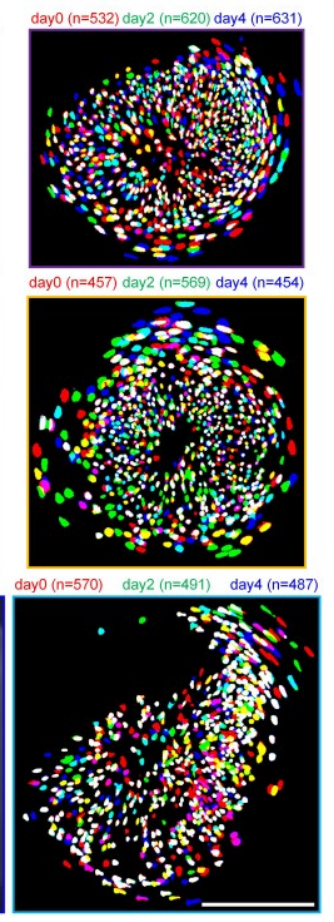

(b)
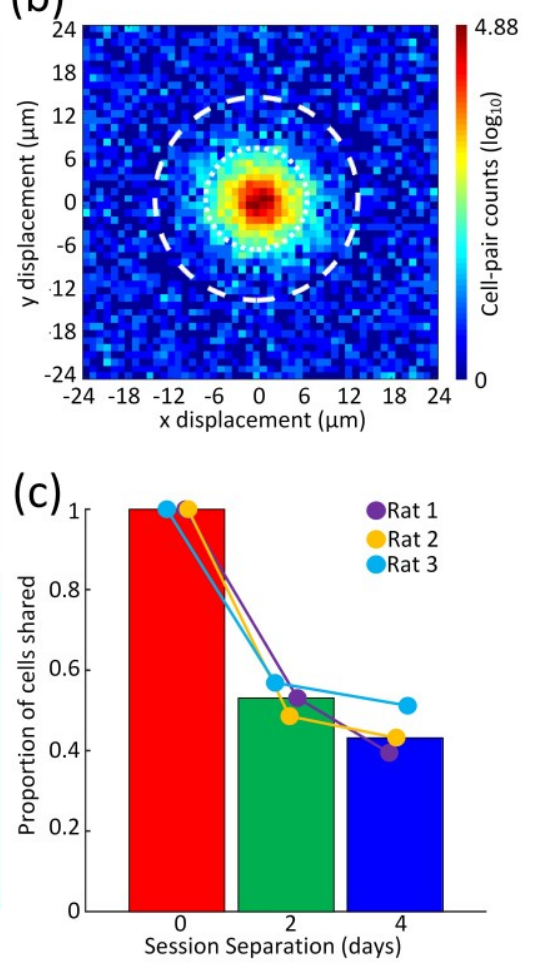

Figure s6. Cells can be reliably recorded across sessions. Three imaging sessions were performed in the same task with 48-hours separating each recording to demonstrate that the MiniLFOV is capable of consistently recording activity from the same population of cells across multiple sessions. (a) Mean frames (Column 1-3) and extracted contours of cells (Column 4) across all three recording days from Rat $1\left(N_{0}=532, N_{2}=620, N_{4}=631\right)$, Rat $2\left(N_{0}=457, N_{2}=569, N_{4}=454\right)$, and Rat $3\left(N_{0}=570, N_{2}=491, N_{4}=487\right)$. The contours of cells are colored by the recording day (day $0=$ red, day $2=$ green, day4=blue). The algorithm to match cells across all sessions is in Methods. Scale bar: $500 \mu \mathrm{m}$. (b) Distribution of centroid displacements for all cell pairs within a 24- $\mu \mathrm{m}$ radius, showing a large population of cell within the same location after across-day alignment. Distributions of centroid distances and spatial correlation for all cell pairs within $24 \mu \mathrm{m}$ were computed, yielding a two-dimensional distribution that can be modeled and given a probability threshold to match cell pairs. Cell pairs with a probability $>0.5$ for both the centroid and correlation distributions were matched (low centroid distance and high spatial correlation), which is expected when recording the same cell. (c) Population overlap (the number of cells shared between sessions) the average population correlation vector between recording sessions decreased as a function of time from Rat 1 (day $0=100 \%$, day $2=53.1 \%$, day $4=39.5 \%$ ), Rat 2 (day0 $=100 \%$, day $2=48.6 \%$, day $4=43.2 \%$ ), and Rat 3 (day $0=100 \%$, day $2=56.9 \%$, day $4=51.2 \%$ ). 

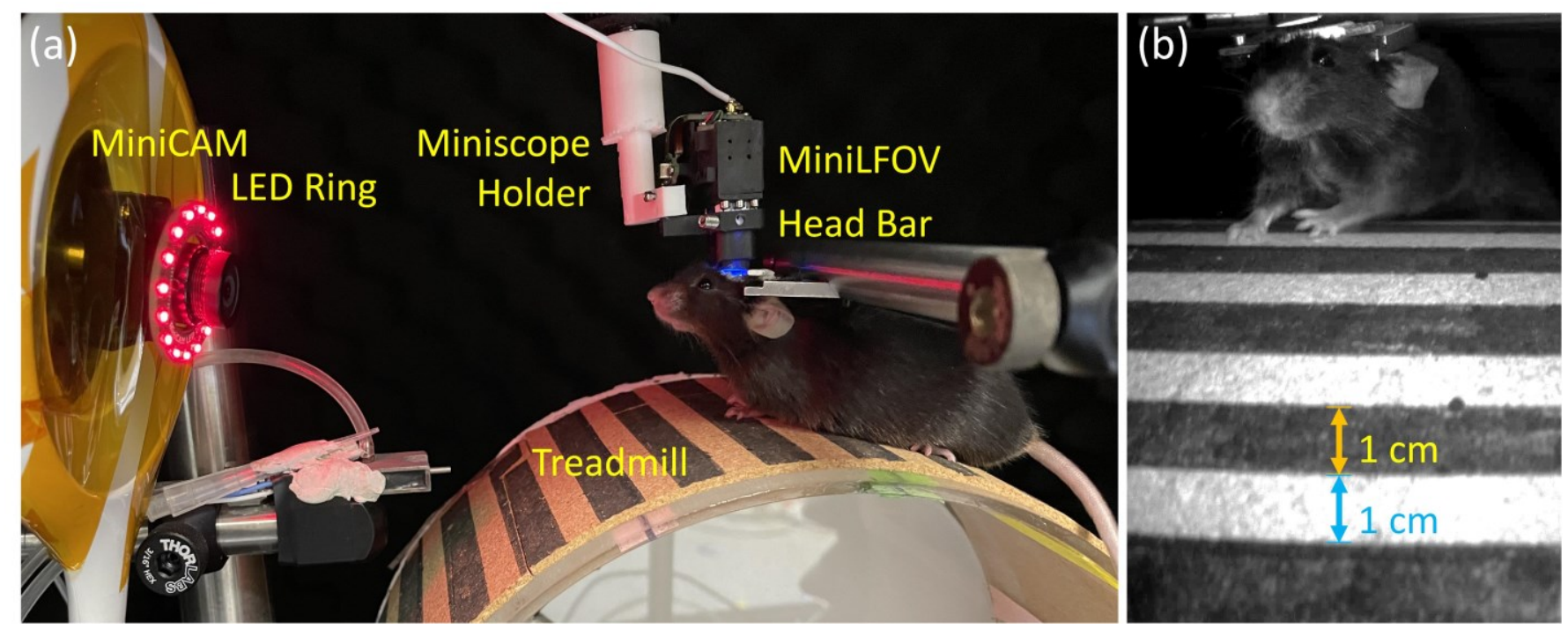

Figure s7. Experimental setup for $\mathrm{Ca}^{2+}$ imaging of dorsal cortex through a cranial window in head-fixed mice. (a) Experimental setup. The mice were head-fixed atop a circular treadmill (22.9-cm diameter and the white and dark bars with 1-cm wide). The MiniLFOV was attached to an articulated ball-head holder mounted on a 3D translation stage built of three linear translation stages (XR25C/M, Thorlabs) so that the lateral position and height of the MiniLFOV relative to the cranial window can be adjusted. A MiniCAM is used to capture the movement speed of the mice and the red LED ring is used for illumination in dark environment. (b) Single frame taken by the MiniCAM. 
(a)
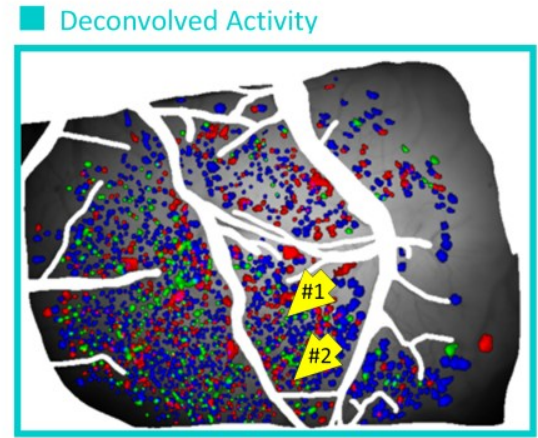

(b)
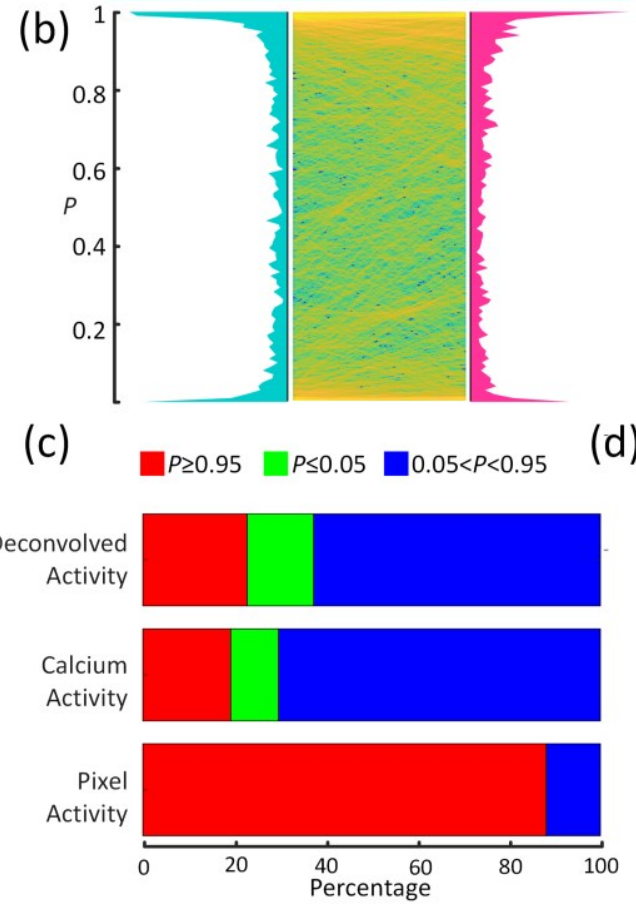

Calcium Activity

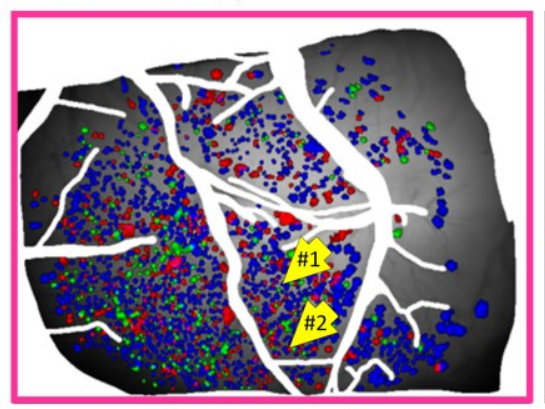

Pixel Activity

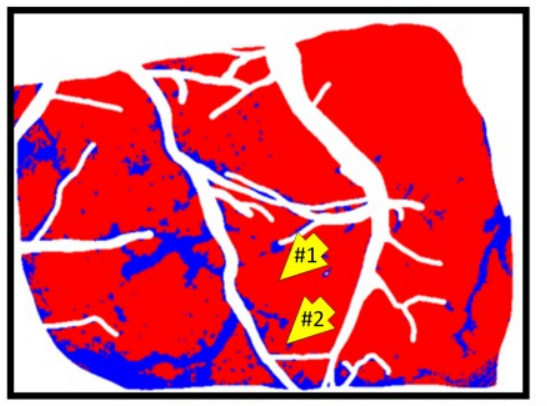

Figure s8. Comparison of neural imaging results with and without using single cell resolution in dorsal cortex in a head-fixed mouse. Movement modulation significance $(P)$ is calculated using the same approach as described in Methods. (a) Color coded cell contours (left and middle) and pixels (right) based on their activity being significantly increased during locomotion (red) or rest (green) with all other cells/pixels shown in blue. Left uses deconvolved neural activity from demixed cells extracted using CalmAn. Middle uses non-deconvolved calcium activity from demixed cells extracted using CalmAn. Right uses $2 \times 2$ mean binned pixel activity $(\sim 10$ $\mu \mathrm{m}$ object size per pixel) from the motion corrected recording approximating results from non-single cell imaging approaches. Scale bar: $500 \mu \mathrm{m}$. (b) Heatmap of projecting the $P$ values of all 1903 cells/pixels from deconvolved activity to calcium activity (left), from calcium activity to pixel activity (middle), and from pixel activity to deconvolved activity (right). Additionally, the histogram of the $P$ values of all cells is shows (deconvolved activity $=$ turquoise, calcium activity = fuchsia, and pixel activity = black). Significance values for cells remain similar for both the deconvolved and calcium activity but almost all shift to very high $P$ values when using pixel activity. (c) Percentage of classified cell types using deconvolved activity ("Locomotion cells": red, N=434, 22.8\%; "other cells": blue, $\mathrm{N}=1193,62.7 \%$; and "resting cells": green, $\mathrm{N}=276,14.5 \%$ ), calcium activity ("Locomotion cells": red, $\mathrm{N}=365,19.180 \%$; "other cells": blue, $\mathrm{N}=1339,70.4 \%$; and "resting cells": green, $\mathrm{N}=199,10.5 \%$ ) and pixel activity ("Locomotion cells": red, $\mathrm{N}=1678,88.2 \%$; "other cells": blue, $\mathrm{N}=225,11.8 \%$; and "resting cells": green, $\mathrm{N}=0,0 \%$ ). Without the ability to demix fluorescence from nearby neurons, cell classification based on activity of center pixel value is not able to resolve any resting cells. (d) Normalized deconvolved activity (turquoise), calcium activity (fuchsia), and center pixel activity (black) of 7-minute recording session from chosen cell \#1 and cell \#2 shown in (a). Shaded gray areas represent the periods of animal locomotion. Cell \#1 labeled as a "resting cell" calculated with deconvolved activity turned to an "other cell" calculated with calcium activity, and labeled as a "locomotion cell" calculated with pixel activity. Cell \#2 is labeled as a "locomotion cell" with all 3 methods. 

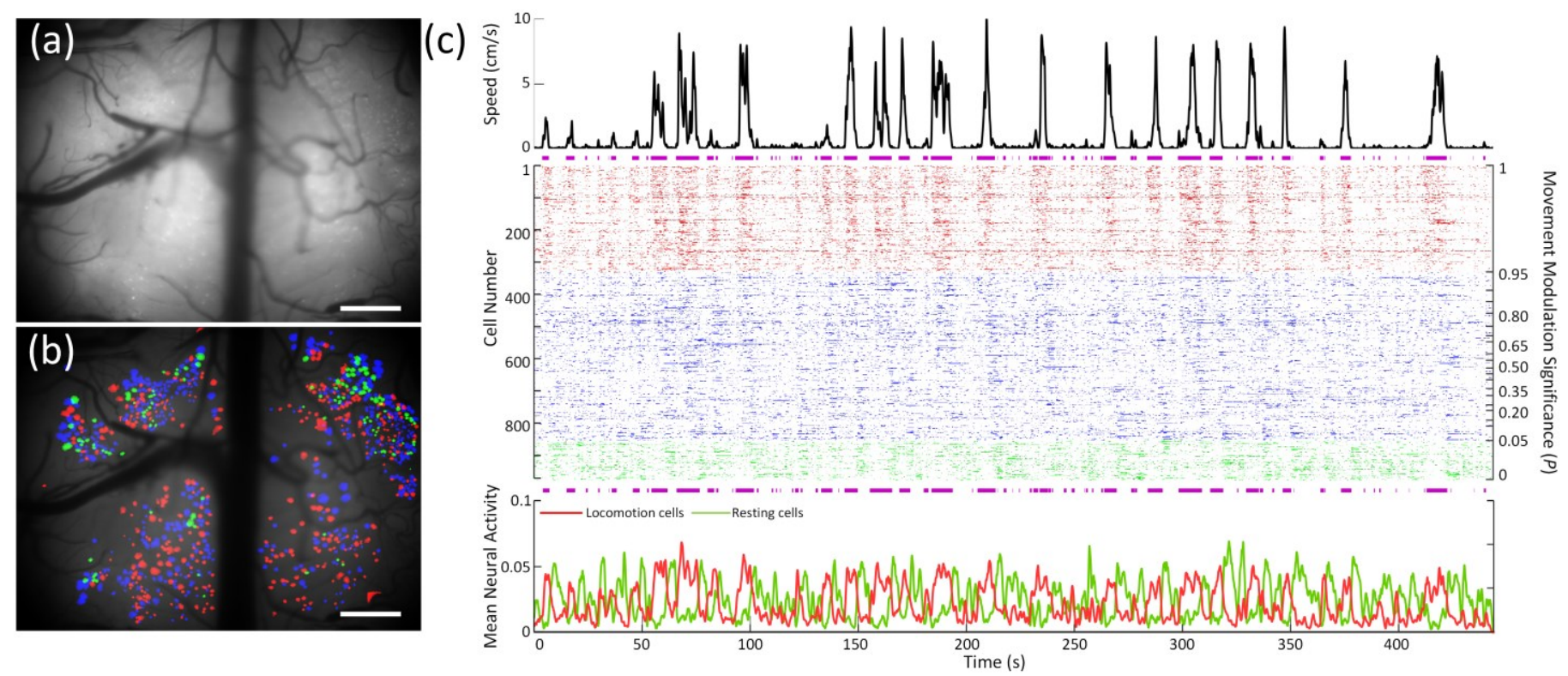

Figure s9. $\mathrm{Ca}^{2+}$ imaging of dorsal cortex through a midcranial window in an additional head-fixed mouse. (a) Maximum projection from a 8-minute recording session after motion correction. Scale bar: $500 \mu \mathrm{m}$. (b) Contours of extracted cells from CNMF-E analysis via CalmAn (colored according based on modulation of neuronal activity during locomotion) overlayed on the minimum projection of the recording session( $\mathrm{N}=971)$. Scale bar: $500 \mu \mathrm{m}$. (c) Relationship between the circular treadmill motion and extracted deconvolved neural activity. (Top) Circular treadmill speed trace. Purple bars under speed trace denote periods of animal locomotion. (Middle) Normalized deconvolved neural activity of all neurons. Neurons are sorted based on the modulation of neuronal activity during period of locomotion. "Locomotion cells": red, N=329, 33.88\%; "other cells": blue, N=520, $53.55 \%$; and "resting cells": green, $\mathrm{N}=122,12.57 \%$. (Bottom) Mean of the normalized deconvolved activity of "locomotion cells" (red) and "resting cells" (green). 
bioRxiv preprint doi: https://doi.org/10.1101/2021.11.21.469394; this version posted November 22, 2021. The copyright holder for this preprint (which was not certified by peer review) is the author/funder, who has granted bioRxiv a license to display the preprint in perpetuity. It is made available under aCC-BY-NC-ND 4.0 International license.

Table 1. Three configurations of MiniLFOV.

\begin{tabular}{|c|c|c|c|c|c|c|c|c|c|c|}
\hline \multirow[b]{2}{*}{ Configurations } & \multirow[b]{2}{*}{$\begin{array}{c}\mathrm{L} \times \mathrm{W} \times \mathrm{H} \\
(\mathrm{mm})\end{array}$} & \multicolumn{9}{|c|}{ Specifications } \\
\hline & & $\begin{array}{c}\text { Weight } \\
\text { (g) }\end{array}$ & $\begin{array}{c}\text { FOV } \\
\left(\mathrm{mm}^{2}\right)\end{array}$ & $\begin{array}{l}\text { Resolution } \\
\qquad(\mu \mathrm{m})\end{array}$ & $\begin{array}{l}\text { WD } \\
(\mathrm{mm})\end{array}$ & M & Pixel & $\begin{array}{c}\text { Frame } \\
\text { rate } \\
\text { (FPS) }\end{array}$ & $\begin{array}{l}\text { Clock } \\
\text { (MHz) }\end{array}$ & Time \\
\hline \multirow[t]{2}{*}{ Wire/WD:1.8 mm } & \multirow{2}{*}{$\begin{array}{c}35 \times 20 \times \\
35\end{array}$} & 14 & $\begin{array}{c}3.1 \times 2 \\
3\end{array}$ & $2.5-4.4$ & 1.8 & 1.86 & $\begin{array}{c}2592 \times \\
1944\end{array}$ & 11 & 66.67 & Long \\
\hline & & 14 & $\begin{array}{c}3.1 \times 2 \\
3\end{array}$ & $4.4-6.2$ & 1.8 & 1.86 & $\begin{array}{c}1296 \times \\
972\end{array}$ & 23 & 66.67 & Long \\
\hline \multirow[t]{2}{*}{ Wire/WD:3.5 mm } & \multirow{2}{*}{$\begin{array}{c}35 \times 20 \times \\
30\end{array}$} & 14 & $\begin{array}{c}3.6 \times 2 \\
7\end{array}$ & $3.5-6.2$ & 3.5 & 1.56 & $\begin{array}{c}2592 \times \\
1944\end{array}$ & 11 & 66.67 & Long \\
\hline & & 14 & $\begin{array}{c}3.6 \times 2 \\
7\end{array}$ & $6.2-7.8$ & 3.5 & 1.56 & $\begin{array}{c}1296 x \\
972\end{array}$ & 23 & 66.67 & Long \\
\hline \multirow{2}{*}{ Wirefree } & & & & & & & & & & \\
\hline & $\begin{array}{c}35 \times 20 \times \\
35 \\
35 \times 20 \times \\
30\end{array}$ & 25 & $\begin{array}{c}1.4 \times 1 \\
4 \\
1.7 \times 1 \\
7\end{array}$ & $\begin{array}{l}4.4-6.2 \\
6.2-7.8\end{array}$ & $\begin{array}{l}1.8 \\
3.5\end{array}$ & $\begin{array}{l}1.86 \\
1.56\end{array}$ & $\begin{array}{c}608 x \\
608\end{array}$ & 20 & 24 & $\begin{array}{l}\leq 1 \\
\text { hour }\end{array}$ \\
\hline
\end{tabular}


bioRxiv preprint doi: https://doi.org/10.1101/2021.11.21.469394; this version posted November $22,2021$. The copyright holder for this preprint (which was not certified by peer review) is the author/funder, who has granted bioRxiv a license to display the preprint in perpetuity. It is made available under aCC-BY-NC-ND 4.0 International license.

Table 2. List of components for MiniLFOV assembly.

\begin{tabular}{|c|c|c|c|c|c|}
\hline & Component & $\begin{array}{l}\text { Qua } \\
\text { ntity }\end{array}$ & Vendor & Part \# & Weblink \\
\hline & Optics & 11 & & & \\
\hline L1 & $\begin{array}{l}6.25 \mathrm{~mm} \text { Dia. } \mathrm{x} \\
\text { 60mm FL, MgF2 } \\
\text { Coated, Achromatic } \\
\text { Doublet Lens }\end{array}$ & 1 & $\begin{array}{l}\text { Edmund } \\
\text { Optics }\end{array}$ & \#45-345 & $\frac{\frac{\text { https://www.edmundoptics.com/p/625m }}{\text { m-dia-x-60mm-fl-mgfsub2sub-coated- }}}{\text { achromatic-doublet-lens/5815/ }}$ \\
\hline L2-1 & $\begin{array}{l}\text { 9mm Diameter x } \\
12 \mathrm{~mm} \text { EFL } \\
\text { Aspherized } \\
\text { Achromatic Lens }\end{array}$ & 1 & $\begin{array}{l}\text { Edmund } \\
\text { Optics }\end{array}$ & \#49-656 & $\frac{\text { https://www.edmundoptics.com/p/9mm- }}{\frac{\text { diameter-x-12mm-efl-aspherized- }}{\text { achromatic-lens/10162/ }}}$ \\
\hline L3-1 & $\begin{array}{l}\text { 9mm Dia. } \times 27 \mathrm{~mm} \\
\text { FL, MgF2 Coated, } \\
\text { Achromatic Doublet } \\
\text { Lens }\end{array}$ & 1 & $\begin{array}{l}\text { Edmund } \\
\text { Optics }\end{array}$ & \#45-092 & $\frac{\text { https://www.edmundoptics.com/p/9mm- }}{\frac{\text { dia-x-27mm-fl-mgfsub2sub-coated- }}{\text { achromatic-doublet-lens } / 5583 /}}$ \\
\hline L2-2 & $\begin{array}{l}\text { 9mm Diameter x } \\
\text { 18mm EFL } \\
\text { Aspherized } \\
\text { Achromatic Lens }\end{array}$ & 1 & $\begin{array}{l}\text { Edmund } \\
\text { Optics }\end{array}$ & \#49-657 & $\frac{\text { https://www.edmundoptics.com/p/9mm- }}{\frac{\text { diameter-x-18mm-efl-aspherized- }}{\text { achromatic-lens/10163/ }}}$ \\
\hline L3-2 & $\begin{array}{l}9 \mathrm{~mm} \text { Dia. } \times 36 \mathrm{~mm} \\
\text { FL, MgF2 Coated, } \\
\text { Achromatic Doublet } \\
\text { Lens }\end{array}$ & 1 & $\begin{array}{l}\text { Edmund } \\
\text { Optics }\end{array}$ & \#45264 & $\frac{\text { https://www.edmundoptics.com/p/9mm- }}{\frac{\text { dia-x-36mm-fl-mgfsub2sub-coated- }}{\underline{\text { achromatic-doublet-lens/5748/ }}}}$ \\
\hline L4 & $\begin{array}{l}\text { 12.5mm Diameter x } \\
14 \mathrm{~mm} \text { EFL } \\
\text { Aspherized } \\
\text { Achromatic Lens }\end{array}$ & 1 & $\begin{array}{l}\text { Edmund } \\
\text { Optics }\end{array}$ & \#49-658 & $\frac{\frac{\text { https://www.edmundoptics.com/p/125m }}{\text { m-diameter-x-14mm-efl-aspherized- }}}{\underline{\text { achromatic-lens/10164/ }}}$ \\
\hline L5 & $\begin{array}{l}\text { 12.0mm Diameter x } \\
-48 \mathrm{FL}, \mathrm{MgF} 2 \\
\text { Coated, Plano- } \\
\text { Concave Lens }\end{array}$ & 1 & $\begin{array}{l}\text { Edmund } \\
\text { Optics }\end{array}$ & \#45-019 & $\frac{\frac{\mathrm{https}: / / \text { www.edmundoptics.com/p/120m }}{\mathrm{m} \text {-diameter-x48-fl-mgfsub2sub-coated- }}}{\text { plano-concave-lens/5531/ }}$ \\
\hline ExF & $4 \times 4 \times 1 \mathrm{~mm}^{3}$ & 1 & Chroma & ET470/40x & $\frac{\text { https://www.chroma.com/products/parts/ }}{\text { et } 470-40 x}$ \\
\hline $\mathrm{DM}$ & $14 \times 10 \times 1 \mathrm{~mm}^{3}$ & 1 & Chroma & T500spxr & Ask for quote \\
\hline $\mathrm{EmF}$ & $10 \times 10 \times 1 \mathrm{~mm}^{3}$ & 1 & Chroma & ET525/50m & $\begin{array}{l}\frac{\text { https://www.chroma.com/products/parts/ }}{\text { et525-50m }} \\
\text { https://www.corning.com/worldwide/en/pr }\end{array}$ \\
\hline EWL & $\begin{array}{l}\text { A-58N with an Anti- } \\
\text { Reflective coating } \\
\text { optimized in the } \\
\text { visible range }\end{array}$ & 1 & $\begin{array}{l}\text { Varioptic } \\
\text { s/Corning }\end{array}$ & $\begin{array}{c}\text { Corning Arctic } \\
58 \mathrm{~N}\end{array}$ & $\begin{array}{l}\frac{\text { oducts/advanced-optics/product- }}{\text { materials/corning-varioptic- }} \\
\frac{\text { lenses/corning varioptic lenses introdu }}{\text { ces new a-58n variable focus liquid- }} \\
\frac{\text { lens.html }}{}\end{array}$ \\
\hline & CMOS Sensor & 1 & & & \\
\hline $\begin{array}{c}\text { CMO } \\
S\end{array}$ & $\begin{array}{l}\text { 1/2.5-Inch } 5 \mathrm{Mp} \\
\text { CMOS } \\
\text { Digital Image } \\
\text { Sensor, Onsemi }\end{array}$ & 1 & Digikey & $\begin{array}{l}488- \\
\text { MT9P031/12 } \\
\text { STM-DP-ND }\end{array}$ & $\begin{array}{l}\frac{\text { https://www.digikey.com/en/products/det }}{\frac{\text { ail/onsemi/MT9P031/12STM- }}{\text { DP/10447064 }}} \\
\frac{\text { https://www.onsemi.com/pdf/datasheet/ }}{\text { mt9p031-d.pdf }}\end{array}$ \\
\hline & Excitation LEDs & 2 & & & \\
\hline & $\begin{array}{l}\text { LED LUXEON Z } \\
\text { BLUE 470NM 2SMD }\end{array}$ & 2 & Digikey & LXZ1-PB01 & $\begin{array}{l}\frac{\text { https://www.digikey.at/product- }}{\text { detail/en/lumileds/LXZ1-PB01/1416- }} \\
\text { 1052-1-ND/3961157 }\end{array}$ \\
\hline & Heac & & & & \\
\hline
\end{tabular}


bioRxiv preprint doi: https://doi.org/10.1101/2021.11.21.469394; this version posted November 22, 2021. The copyright holder for this preprint

(which was not certified by peer review) is the author/funder, who has granted bioRxiv a license to display the preprint in perpetuity. It is made available under aCC-BY-NC-ND 4.0 International license.

\begin{tabular}{|c|c|c|c|c|}
\hline $\begin{array}{l}\text { Accelerometer, } \\
\text { Gyroscope, } \\
\text { Magnetometer, } 9 \\
\text { Axis Sensor } I^{2} \mathrm{C}, \\
\text { UART Output }\end{array}$ & 1 & Digikey & $\begin{array}{l}\text { 828-1058-1- } \\
\text { ND }\end{array}$ & $\frac{\text { https://www.digikey.com/en/products/det }}{\text { ail/bosch-sensortec/BNO055/6136301 }}$ \\
\hline \multicolumn{5}{|l|}{ 3D Printer } \\
\hline $\begin{array}{l}\text { High-end Stereolithographic } \\
\text { (SLA) printer }\end{array}$ & 1 & Formlabs & Form 3 & https://formlabs.com/3d-printers/form-3/ \\
\hline \multicolumn{5}{|l|}{ Housing } \\
\hline Objective module body & 1 & $\mathrm{~N} / \mathrm{A}$ & $\mathrm{N} / \mathrm{A}$ & $\begin{array}{l}\text { https://github.com/Aharoni- } \\
\text { Lab/Miniscope-LFOV/blob/main/3D- } \\
\text { Printed-Bodies MiniLFOV/Objective- } \\
\text { Module Body MiniLFOV.stl }\end{array}$ \\
\hline Spacer & 1 & $\mathrm{~N} / \mathrm{A}$ & $\mathrm{N} / \mathrm{A}$ & $\begin{array}{l}\text { https://github.com/Aharoni- } \\
\text { Lab/Miniscope-LFOV/blob/main/3D- } \\
\frac{\text { Printed-Bodies MiniLFOV/Objective- }}{\text { Spacer MiniLFOV.stl }}\end{array}$ \\
\hline Emission module body & 1 & $\mathrm{~N} / \mathrm{A}$ & $\mathrm{N} / \mathrm{A}$ & $\begin{array}{l}\text { https://github.com/Aharoni- } \\
\frac{\text { Lab/Miniscope-LFOV/blob/main/3D- }}{\text { Printed-Bodies MiniLFOV/Emission- }} \\
\text { Module-Body MiniLFOV.stl }\end{array}$ \\
\hline Cover & 1 & $\mathrm{~N} / \mathrm{A}$ & $\mathrm{N} / \mathrm{A}$ & $\begin{array}{l}\frac{\overline{\text { https://github.com/Aharoni- }}}{\text { Lab/Miniscope-LFOV/blob/main/3D- }} \\
\text { Bodies MiniLFOV/Cover MiniLFOV.stl }\end{array}$ \\
\hline Cable Holder & 1 & $N / A$ & $\mathrm{~N} / \mathrm{A}$ & $\begin{array}{c}\frac{\text { https://github.com/Aharoni- }}{\text { Lab/Miniscope-LFOV/blob/main/3D- }} \\
\frac{\text { Printed-Bodies MiniLFOV/Cable- }}{\text { Holder MiniLFOV.stl }}\end{array}$ \\
\hline L5 Lens Holder & 1 & $\mathrm{~N} / \mathrm{A}$ & $\mathrm{N} / \mathrm{A}$ & $\begin{array}{c}\frac{\text { https://github.com/Aharoni- }}{\text { Lab/Miniscope-LFOV/blob/main/3D- }} \\
\frac{\text { Printed-Bodies MiniLFOV/L5- }}{\text { Holder MiniLFOV.stl }}\end{array}$ \\
\hline CMOS module body & 1 & $\mathrm{~N} / \mathrm{A}$ & $\mathrm{N} / \mathrm{A}$ & $\begin{array}{c}\text { https://github.com/Aharoni- } \\
\frac{\text { Lab/Miniscope-LFOV/blob/main/3D- }}{\text { Printed-Bodies MiniLFOV/CMOS- }} \\
\text { Module-Body MiniLFOV.stl }\end{array}$ \\
\hline \multicolumn{5}{|l|}{ Circuits } \\
\hline MiniLFOV Circuit & 1 & $\mathrm{~N} / \mathrm{A}$ & $\mathrm{N} / \mathrm{A}$ & $\frac{\text { https://github.com/Aharoni- }}{\text { Lab/Miniscope-LFOV }}$ \\
\hline \multicolumn{5}{|l|}{ Screws } \\
\hline M1 thread-forming screws & 12 & $\begin{array}{l}\text { McMaste } \\
\text { r-Carr }\end{array}$ & $96817 a 704$ & $\frac{\text { https://www.mcmaster.com/tapping- }}{\text { screws/screw-size } 1 /}$ \\
\hline $\begin{array}{c}\text { 18-8 Stainless Steel Socket } \\
\text { Head Screw, 0-80 Thread } \\
\text { Size }\end{array}$ & 8 & $\begin{array}{l}\text { McMaste } \\
\text { r-Carr }\end{array}$ & 92196A052 & $\frac{\text { https://www.mcmaster.com/catalog/9219 }}{\underline{6 a 052}}$ \\
\hline $\begin{array}{l}\text { 4-40 Stainless Steel } \\
\text { Setscrew, 1/4" Long }\end{array}$ & 2 & Thorlabs & SS4S025 & $\frac{\text { https://www.thorlabs.com/thorproduct.cf }}{\text { m?partnumber=SS4S025 }}$ \\
\hline $\begin{array}{l}\text { Cabling \& Hardware } \\
\text { Control }\end{array}$ & 1 & & & \\
\hline $50 \Omega$, flexible coaxial cable & 1 & $\begin{array}{l}\text { Cooner } \\
\text { Wire }\end{array}$ & $\begin{array}{l}\text { CW2040- } \\
3650 S R\end{array}$ & $\frac{\text { https://www.coonerwire.com/mini-coax/ }}{\text { https://open-ephys.org/miniscope- }}$ \\
\hline $\begin{array}{l}\text { Data acquisition (DAQ) } \\
\text { device }\end{array}$ & 1 & $\begin{array}{l}\text { Open } \\
\text { Ephys }\end{array}$ & DAQ v3.3 & $\underline{\text { http://miniscope.org/index.php/Main Pag }}$ \\
\hline
\end{tabular}


bioRxiv preprint doi: https://doi.org/10.1101/2021.11.21.469394; this version posted November 22, 2021. The copyright holder for this preprint (which was not certified by peer review) is the author/funder, who has granted bioRxiv a license to display the preprint in perpetuity. It is made available under aCC-BY-NC-ND 4.0 International license.

\begin{tabular}{|c|c|c|c|c|}
\hline Wire-free DAQ & 1 & N/A & N/A & $\frac{\text { https://github.com/Aharoni- }}{\text { Lab/Miniscope-LFOV }}$ \\
\hline $\begin{array}{c}\text { SparkFun Infrared Control } \\
\text { Kit }\end{array}$ & 1 & Mouser & $\begin{array}{l}\text { 474-KIT- } \\
14677 \\
\text { KIT-14677 }\end{array}$ & 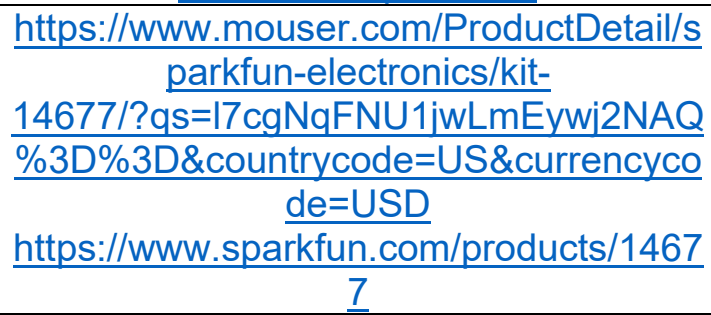 \\
\hline $\begin{array}{l}\text { EMU FOR SAM AND } \\
\text { AVR MCU KIT }\end{array}$ & 1 & Digikey & $\begin{array}{l}\text { ATATMEL- } \\
\text { ICE-ND }\end{array}$ & $\frac{\text { https://www.digikey.com/en/products/det }}{\frac{\text { ail/microchip-technology/ATATMEL- }}{\text { ICE/4753379 }}}$ \\
\hline \multicolumn{5}{|l|}{ Firmware \& Software } \\
\hline $\begin{array}{l}\text { DAQ firmware for UCLA } \\
\text { Miniscope platforms }\end{array}$ & $\mathrm{N} / \mathrm{A}$ & $\mathrm{N} / \mathrm{A}$ & $\mathrm{N} / \mathrm{A}$ & $\begin{array}{l}\text { https://github.com/Aharoni- } \\
\text { Lab/Miniscope-DAQ-Cypress-firmware }\end{array}$ \\
\hline $\begin{array}{l}\text { Aharoni-Lab } \\
\text { Miniscope-DAQ-QT- } \\
\text { Software }\end{array}$ & N/A & N/A & v1.11 & $\underline{\underline{\text { hab/Miniscope-DAQ-QT-Software }}}$ \\
\hline Wire-free DAQ firmware & $\mathrm{N} / \mathrm{A}$ & N/A & $\mathrm{N} / \mathrm{A}$ & $\begin{array}{c}\frac{\text { https://github.com/Aharoni- }}{\text { Lab/Miniscope-Wire-Free- }} \\
\text { DAQ/tree/master/MCU-firmware }\end{array}$ \\
\hline$\frac{\text { microSD configuration }}{\text { MiniCAM }}$ & $\mathrm{N} / \mathrm{A}$ & $\mathrm{N} / \mathrm{A}$ & $\mathrm{N} / \mathrm{A}$ & https://mh-nexus.de/en/hxd/ \\
\hline $\begin{array}{c}\text { Open-source behavioral } \\
\text { camera for imaging freely } \\
\text { behaving animals in } \\
\text { behavioral and neuroscience } \\
\text { experiments }\end{array}$ & 1 & $\begin{array}{l}\text { Open } \\
\text { Ephys }\end{array}$ & N/A & $\frac{\text { https://github.com/Aharoni-Lab/MiniCAM }}{\underline{\text { https://open-ephys.org/minicam/minicam }}}$ \\
\hline \multicolumn{5}{|l|}{ Piplines } \\
\hline CalmAn & N/A & N/A & N/A & $\frac{\text { https://github.com/flatironinstitute/CalmA }}{\underline{n}}$ \\
\hline OASIS & $\mathrm{N} / \mathrm{A}$ & N/A & $\mathrm{N} / \mathrm{A}$ & $\frac{\text { https://github.com/zhoupc/OASIS matla }}{\underline{\mathrm{b}}}$ \\
\hline $\begin{array}{l}\text { Wire-free Data read from } \\
\text { microSD }\end{array}$ & N/A & $\mathrm{N} / \mathrm{A}$ & N/A & $\begin{array}{l}\frac{\underline{\text { https://github.com/Aharoni- }}}{\text { Lab/Miniscope-Wire-Free- }} \\
\text { DAQ/tree/master/Wire-Free-DAQ- } \\
\text { Software }\end{array}$ \\
\hline
\end{tabular}

